\title{
Asymptotic dynamics on the worldline for spinning particles
}

\author{
Domenico Bonocore \\ Institut für Theoretische Physik, Westfälische Wilhelms-Universität Münster, \\ Wilhelm-Klemm-Straße 9, D-48149 Münster, Germany \\ E-mail: domenico.bonocore@uni-muenster.de
}

AbStRact: There has been a renewed interest in the description of dressed asymptotic states à la Faddeev-Kulish. In this regard, a worldline representation for asymptotic states dressed by radiation at subleading power in the soft expansion, known as the Generalized Wilson Line (GWL) in the literature, has been available for some time, and it recently found applications in the derivation of factorization theorems for scattering processes of phenomenological relevance. In this paper we revisit the derivation of the GWL in the light of the well-known supersymmetric wordline formalism for the relativistic spinning particle. In particular, we discuss the importance of wordline supersymmetry to understand the contribution of the soft background field to the asymptotic dynamics. We also provide a derivation of the GWL for the gluon case, which was not previously available in the literature, thus extending the exponentiation of next-to-soft gauge boson corrections to Yang-Mills theory. Finally, we comment about possible applications in the current research about asymptotic states in scattering amplitudes for gauge and gravity theories and their classical limit.

KeYwords: Perturbative QCD, Resummation, Scattering Amplitudes

ArXiv EPrint: 2009.07863 


\section{Contents}

1 Introduction 1

2 Spin zero $\quad 5$

2.1 Dressed propagators and conserved charges 5

2.2 Asymptotic propagators in a gauge boson background 8

3 Spin one-half $\quad 10$

$\begin{array}{lll}3.1 & \text { Worldline representation } & 10\end{array}$

\begin{tabular}{ll}
3.2 & Numerator contribution \\
\hline
\end{tabular}

$\begin{array}{ll}3.3 & \text { Denominator contribution } \\ \end{array}$

4 Spin one $\quad 16$

4.1 Generalized Wilson Line for gluons 16

$\begin{array}{ll}4.2 \text { The supersymmetric model } & 19\end{array}$

5 Discussion

\section{Introduction}

The use of first-quantized techniques in quantum field theories has a long history. The origin can be traced back to the early days of quantum electrodynamics, when Schwinger [1] proposed to use the distribution identity

$$
\lim _{\epsilon \rightarrow 0^{+}} \frac{i}{H+i \epsilon}=\lim _{\epsilon \rightarrow 0^{+}} \int_{0}^{\infty} d T e^{i(H+i \epsilon) T}
$$

to interpret the inverse propagator of a scalar field as the matrix elements of a Hamiltonian $H\left(\hat{x}_{\mu}, \hat{p}_{\mu}\right)$ governing the evolution in proper time $T$ of a first-quantized model with canonical variables $\hat{x}_{\mu}$ and $\hat{p}_{\mu}$. By writing a path integral representation for the matrix elements of the evolution operator $e^{i H T}$, the dynamics of the relativistic particle then is described by a classical action for the fields $x^{\mu}(t)$ and $p^{\mu}(t)$ leaving in a one dimensional space of length $T$. The worldline formalism $[2,3]$ is based on the observation that such actions can be derived from first principles, starting from the constrained quantization of the relativistic particle rather than the field theory propagator.

A major obstacle for the program is given by the presence of matrices in the exponent, which occur e.g. for spinning or colored particles. This in general requires either a path ordering prescription or the introduction of Grassman variables $\psi_{\mu}(t)$. With the latter option, the action for a free massless particle of spin $N / 2$ reads [4-7]

$$
S=\int d t\left(p_{\mu} \dot{x}^{\mu}+\frac{i}{2} \psi_{i}^{\mu} \dot{\psi}_{\mu}^{i}-\frac{1}{2} e p_{\mu} p^{\mu}-i \chi_{i} \psi_{\mu}^{i} p^{\mu}-\frac{i}{2} a_{i j} \psi_{i}^{\mu} \psi_{\mu}^{j}\right)
$$


with $i=1, \ldots, N$. The symmetry structure of this action is quite rich, since it involves reparametrization invariance, $N$-extended local supersymmetry and $O(N)$ gauge invariance, with the corresponding gauge fields $e(t), \chi_{i}(t)$ and $a_{i j}(t)$. As is evident from eq. (1.2), these fields play the role of Lagrange multipliers which are typically gauged away for practical calculations.

Most of the early work (see e.g. [8-11]) focused on quantization issues of the supersymmetric model and on attempts to formulate it on curved spacetime. However, an application showing the practical advantages of this formulation for actual calculations remained somewhat elusive. In this regard, a major advance was put forward by Strassler [12]. The idea stems from the fact that a one-loop effective action in the background of some gauge boson field can be described as a dressed propagator whose extrema have been closed to form a loop. Using a worldline representation for such a propagator, Strassler verified that upon solving the path integral order by order in the coupling constant $g$, one is left at order $g^{N}$ with the amplitude for $N$ external gauge bosons in terms of integrals over Feynman parameters only, thus bypassing the construction of the Feynman amplitude via standard Feynman rules. In this way he recovered the Bern-Kosower rules [13] previously derived from string theory insights.

Motivated by this success, most of the applications of the worldline approach have aimed at the computation of effective actions (see e.g. [14, 15]). On the other hand, the worldline representation of an open dressed propagator has received less attention, with intermittent progress [16-25], although it has a richer structure that might reveal new methods for the efficient calculation of scattering amplitudes. ${ }^{1}$

A situation which is somewhat intermediate between the open and the closed dressed propagators is given by asymptotic dressed propagators. This is what was considered by Laenen, White and Stavenga [28], who combined the aferomentioned body of work and the long-standing problem of infrared exponentiation beyond the leading power in the soft expansion (there dubbed next-to-eikonal) [29-32]. Such exponentiation emerges neatly in this picture by solving the path integral representation of the dressed propagator order by order in the soft expansion (but to all-orders in the coupling constant). More specifically, in the abelian case it corresponds to the familiar exponentiation of connected diagrams, while for non-abelian theories one has to invoke the so-called "replica trick" from statistical physics. This bypasses highly non-trivial combinatorics that one should use in a purely diagrammatic approach [33], showing the power of the worldline formulation. The approach has also been implemented for soft gravitons in [34].

Building on this work, and motivated by the rising interest in the field of next-toleading power (NLP) corrections to the soft and collinear limits both in phenomenology [35-53] and in more formal contexts [54-70], the worldline description of [28] has proved to be a valuable tool to derive factorization theorems at NLP [71, 72]. The asymptotic dressed propagator defined in this way at NLP has been dubbed Generalized Wilson Line (GWL), and it is defined for a semi-infinite straight line starting from the origin in the

\footnotetext{
${ }^{1}$ For recent progress in this direction see [26, 27].
} 
direction $n^{\mu}$ as

$$
\begin{aligned}
\widetilde{W}_{n}(0, \infty)= & \mathcal{P} \exp \left[g \int \frac{d^{d} k}{(2 \pi)^{d}} \tilde{A}_{\mu}(k)\left(-\frac{n^{\mu}}{n \cdot k}+\frac{k^{\mu}}{2 n \cdot k}-k^{2} \frac{n^{\mu}}{2(n \cdot k)^{2}}-\frac{\mathrm{i} k_{\nu} J^{\nu \mu}}{n \cdot k}\right)\right. \\
& +g^{2} \int \frac{d^{d} k}{(2 \pi)^{d}} \int \frac{d^{d} l}{(2 \pi)^{d}} \tilde{A}_{\mu}(k) \tilde{A}_{\nu}(l)\left(\frac{\eta^{\mu \nu}}{2 n \cdot(k+l)}-\frac{n^{\nu} l^{\mu} n \cdot k+n^{\mu} k^{\nu} n \cdot l}{2(n \cdot l)(n \cdot k)[n \cdot(k+l)]}\right. \\
& \left.\left.+\frac{(k \cdot l) n^{\mu} n^{\nu}}{2(n \cdot l)(n \cdot k)[n \cdot(k+l)]}-\frac{\mathrm{i} J^{\mu \nu}}{n \cdot(k+l)}\right)\right],
\end{aligned}
$$

where $\mathcal{P}$ is the path-ordering symbol and $\tilde{A}_{\mu}(k)=\tilde{A}_{\mu}^{a}(k) T^{a}$ is the Fourier transform of the non-abelian gauge field corresponding to a soft gluon emission of momentum $k^{\mu}$. The term of order $k^{-1}$ in the first line of eq. (1.3) corresponds to the usual Wilson line for a semi-infinite straight path, while the remaining terms correspond to NLP corrections. Two of these subleading terms contain the total angular momentum $J^{\mu \nu}$, which for an emitting particle in the representation specified by the indices $i$ and $j$ is given by the sum of the Lorentz generators for spin $\left(S^{\mu \nu}\right)^{i j}$ and angular momentum $L^{\mu \nu} \delta^{i j}{ }^{2}$

The validity of eq. (1.3) for generic spin has been motivated by the result one would expect for the closed propagator of the one-loop effective action [12, 21], where the coupling between the spin and the background field is contained exclusively in the chromo-magnetic interaction $S_{\mu \nu} F^{\mu \nu}$. In fact, after replacing the external lines of a scattering amplitude with the straight and semi-infinite GWLs, these close at infinity at the cross-section level, by unitarity. Therefore, one should expect the one-loop effective action description to be equivalent when the formalism is implemented in physical processes. Moreover, this picture has been corroborated for tree-level amplitudes with a single soft emission by the recently discovered next-to-soft theorems [55], and a comparison has been provided in [73].

However, one would like a less heuristic argument that could put eq. (1.3) on a solid ground for a generic scattering amplitude. In fact, a derivation of eq. (1.3) from the worldline representation of an open propagator of generic spin is still missing. Even for the Dirac case, which was analyzed in [28], some issues still remain to be clarified. For instance, in that case eq. (1.3) has been obtained by first decomposing the dressed Dirac propagator in terms of the covariant derivative $D_{\mu}=\partial_{\mu}-A_{\mu}$ as $^{3}$

$$
\frac{1}{i \not D-m}=\frac{i \not D+m}{-\not D^{2}-m^{2}}=\frac{i \not D+m}{-D^{2}-m^{2}+S^{\mu \nu} F_{\mu \nu}}
$$

with $S^{\mu \nu}=\frac{i}{4}\left[\gamma^{\mu}, \gamma^{\nu}\right]$, and then writing a worldline representation for the denominator only. In fact, the numerator does not contribute for the one-loop effective action [12]. On the other hand, as remarked in [28], for an asymptotic dressed propagator the numerator does

\footnotetext{
${ }^{2}$ The angular momentum $L^{\mu \nu}$ was not included in the definition provided in $[28,71,72]$, since the corresponding internal emissions from Low's theorem give rise to contributions that do not exponentiate to all-orders, unlike the other terms which are due to external emissions. However, as observed in [72], the separation between internal and external emissions is not gauge invariant. Hence, in order for the Generalized Wilson Line to transform better under gauge tranformations, it might be convenient to include the angular momentum in the definition.

${ }^{3}$ Unlike [28], we use the $(+,-,-,-)$ metric throughout.
} 
contribute but it is supposed to cancel à la LSZ with the numerator of the free inverse propagator. However, this is obviously correct only if the gauge field contribution in the numerator vanishes. What is the mechanism behind that?

Things get even more subtle for spin-one (which was not discussed in [28]), where in the massless case one has to deal with the gauge dependence of the emitting particle. In Feynman gauge, the numerator of the dressed propagator is unity and thus one expects the argument to mimic what done in the case of the one-loop effective action. However, a more precise derivation would be desirable. Moreover, one would like to extend the validity of eq. (1.3) also to massive vector particles, and possibly to higher spin.

Quite generally, what is missing is a clear relation between the supersymmetric formulation of the relativistic spinning particle (i.e. the equivalent of eq. (1.2) with a background field) and the generalized Wilson line of eq. (1.3). This is the main goal of this paper and in fact it will turn out that a clear relation between the two descriptions will answer the previous questions, putting the derivation of the GWL on a firm basis also for spinning particles.

As we have remarked, the need for a clear derivation of the GWL for the case of spinning particle is mainly of phenomenological origin, since QCD scattering processes involve quarks and gluons in the initial and final states. In this regard, the GWL turned out to be useful in the recent attempts to extend the traditional soft-gluon resummation program to NLP. In particular, the GWL has been already implemented in the Leading-Logarithmic NLP soft-gluon resummation for the inclusive production of color-singlet states, such as Higgs production via gluon-fusion [72], although a formal proof from first principles for the spinone case has been lacking. This last point makes a derivation of the spinning GWL from first principles even more desirable.

However, the GWL have a much broader scope, which offers other motivations for the present study. One arises from a revived interest in asymptotic states à la FaddeevKulish [74-87]. Most of these methods revolve around the existence of an asymptotic Hamiltonian governing the evolution of the asymptotic states. In particular, reference $[88,89]$, building on insights from soft-collinear effective theories, provides a systematic calculation of such Hamiltonians that makes the S-matrix infrared finite. The use of GWLs in this context would easily extend such description beyond the leading power in the soft expansion. Indeed, this could provide more efficient definitions of infrared-finite S-matrices, allowing to move effects from the asymptotic to the S-matrix. To this end, it is therefore desirable to have a firm derivation of the GWL also for spinning particles.

More generally, there has been recently a great deal of interest in the classical limits of scattering amplitudes [90-92], mainly motivated by the growing interest in precision calculations in gravitational physics. In particular, there is evidence that the high-energy-limit and the corresponding eikonal approximation are key to extract the classical limit [93-97]. In this context, the semiclassical picture of the GWL extends the eikonal approximation to subleading power, and thus provides a new tool to study the classical limit of scattering amplitudes. Work in this direction has been done in the scalar case in [98, 99]. Besides, the GWL might provide an efficient way to extend the classical limit of soft theorems to subleading power $[68,69,100]$. Therefore, given the importance of spin in gravitational 
physics [101-111], it would be desirable to extend the derivation of the GWL to particles of arbitrary spin.

The structure of the paper is the following. We begin in section 2 by revisiting the scalar case originally presented in [28]. The goal here is to highlight the relation with the worldline formalism and its symmetries, stressing the distinctive features that arise for asymptotic propagators dressed by soft radiation. Then, in section 3 we will move to the Dirac case, where the supersymmetry of the model will allow us to write the dressed propagator in terms of conserved charges and subsequently to observe that the soft field in the numerator does not contribute in the asymptotic limit. Finally, in section 4 we will discuss the spin-one case, where we will first justify the definition of the GWL for gluons without wordline fermions. Then we will discuss the corresponding supersymmetric model, paving the way for a generalization to particles of higher spin. We conclude in section 5 with a short discussion.

\section{Spin zero}

Although the generalized Wilson line for a scalar particle has been already discussed in [28], it is useful to revisit the derivation in a different approach, i.e. starting from the constrained quantization of the relativistic particle, which is more standard in the worldline literature, highlighting the distinctive features that appear in the case of an asymptotic propagator dressed by soft radiation.

\subsection{Dressed propagators and conserved charges}

We start from the well-known ${ }^{4}$ phase space action for a free relativistic scalar particle

$$
S=\int d t\left(p \cdot \dot{x}-e \frac{1}{2}\left(p^{2}-m^{2}\right)\right) .
$$

The system is invariant under the following gauge transformations

$$
\delta x_{\mu}=\xi p^{\mu}, \quad \delta p_{\mu}=0, \quad \delta e=\dot{\xi}
$$

generated by the first-class constraint

$$
Q_{0} \equiv \frac{1}{2}\left(p^{2}-m^{2}\right)
$$

Following the Dirac procedure, and equipped with the Hamiltonian $H=e Q_{0}$, the quantization consists in defining the Hilbert space as the linear space spanned by $|x\rangle$ or $|p\rangle$ where the physical states $|\psi\rangle$ satisfy $Q_{0}|\psi\rangle=0$.

In this language, the free Feynman propagator $\left\langle\phi\left(x_{f}\right) \phi\left(x_{i}\right)\right\rangle$ of the corresponding scalar field $\phi(x)$ can be defined by the matrix elements of the first-quantized operator $\left(2 Q_{0}+i \epsilon\right)^{-1}$, with the following path integral representation

$$
\left\langle\phi\left(x_{f}\right) \phi\left(x_{i}\right)\right\rangle \equiv\left\langle x_{f}\left|\left(2 Q_{0}+i \epsilon\right)^{-1}\right| x_{i}\right\rangle=\frac{1}{2} \int_{x(0)=x_{i}}^{x(1)=x_{f}} \mathcal{D} e \mathcal{D} x \mathcal{D} p e^{-i \int_{0}^{1} d t\left(p \cdot \dot{x}-e\left(Q_{0}+i \epsilon\right)\right)},
$$

\footnotetext{
${ }^{4}$ See e.g. [112] for a pedagogical review.
} 


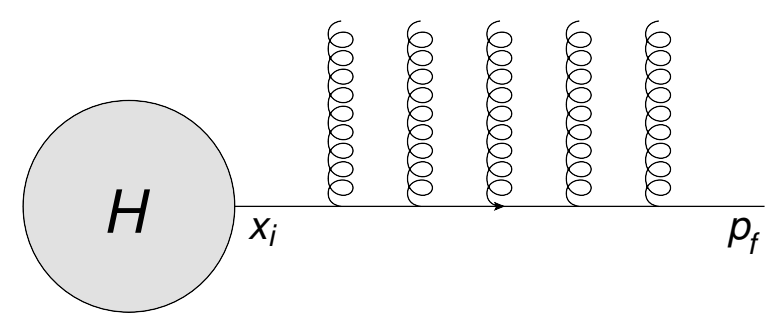

Figure 1. Schematic depiction of a propagator dressed by soft radiation, from an initial state localized at position $x_{i}$ in the Hard function $H$ to a final state of momentum $p_{f}$.

where the integration measures have been normalized to unity. The validity of eq. (2.4) is perhaps clearer after gauge fixing $e(t)=T$. Thanks to $\delta e / \delta \xi$ in eq. (2.2) being fieldindependent, this choice yields a trivial Faddeev-Popov determinant. Then, the path integral over the einbein $e(t)$ reduces to an integration over gauge-non-equivalent parametrizations labeled by the Schwinger proper time $T$. After rescaling $t \rightarrow T t$ in the action, the r.h.s. of eq. (2.4) becomes

$$
\frac{1}{2} \int_{0}^{\infty} d T \int_{x(0)=x_{i}}^{x(T)=x_{f}} \mathcal{D} x \mathcal{D} p e^{-i \int_{0}^{T} d t\left(p \cdot \dot{x}-Q_{0}-i \epsilon\right)}=\frac{1}{2} \int_{0}^{\infty} d T\left\langle x_{f}\left|e^{i\left(Q_{0}+i \epsilon\right) T}\right| x_{i}\right\rangle
$$

which matches $\left\langle x_{f}\left|\left(2 Q_{0}+i \epsilon\right)^{-1}\right| x_{i}\right\rangle$ by virtue of eq. (1.1).

The procedure above can be easily generalized to a scalar particle propagating in a classical background $B(x)$. In that case one has to isolate the quadratic part in the field theory Lagrangian $\mathcal{L}^{(2)}=\phi^{*}(x) \Delta\left(x_{\mu}, \partial_{\mu}\right) \phi(x)$. Then, the corresponding first-quantized system exhibits again a gauge symmetry generated by the Noether charge

$$
Q_{0}^{B}(\hat{x}, \hat{p}) \equiv \frac{1}{2} \Delta\left(\hat{x}_{\mu},-i \hat{p}_{\mu}\right) .
$$

Thus, the definition in eq. (2.4) is still valid, after replacing $Q_{0} \rightarrow Q_{0}^{B}$ in Weyl-ordered form.

So far we have considered a dressed propagator between an initial and a final spacetime points $x_{i}$ and $x_{f}$, since this corresponds to the two-point function $\left\langle\phi\left(x_{f}\right) \phi\left(x_{i}\right)\right\rangle$ in the common second-quantized approach. On the other hand, in the first-quantized approach nothing prevents us from working in a mixed position-momentum representation. For instance, we can consider

$$
\begin{aligned}
\left\langle p_{f}\left|\left(2 Q_{0}^{B}+i \epsilon\right)^{-1}\right| x_{i}\right\rangle & =\frac{1}{2} \int_{0}^{\infty} d T\left\langle p_{f}\left|e^{-i\left(Q_{0}^{B}(\hat{x}, \hat{p})+i \epsilon\right) T}\right| x_{i}\right\rangle \\
& =\frac{1}{2} \int_{0}^{\infty} d T \int_{x(0)=x_{i}}^{p(T)=p_{f}} \mathcal{D} x \mathcal{D} p e^{i p(T) \cdot x(T)-i \int_{0}^{T} d t\left(p \cdot \dot{x}-Q_{0}^{B}(x, p)-i \epsilon\right),}
\end{aligned}
$$

where the uncommon term $p(T) \cdot x(T)$ is due to these mixed boundary conditions. This is the representation used in [28] for asymptotic propagators of a scattering amplitude. Indeed, as depicted in figure 1, in this case one typically considers a dressed propagator emitted from the hard function at a spacetime point $x_{i}^{\mu}$, which will be integrated over, to a final state of momentum $p_{f}^{\mu}$. The reason for having a final state with well-defined 
momentum is that eventually we would like to perform the path integral over $x^{\mu}$ order by order in the soft expansion w.r.t. the hard momentum $p_{f}^{\mu}$.

The previous arguments become clear after expanding around the classical solutions

$$
p^{\mu}(t)=p_{f}^{\mu}+\tilde{p}^{\mu}(t), \quad x^{\mu}(t)=x_{i}^{\mu}+p_{f}^{\mu} t+\tilde{x}^{\mu}(t) .
$$

Then, the dressed propagator reads

$$
\frac{\left\langle p_{f}\left|\left(2 Q_{0}^{B}+i \epsilon\right)^{-1}\right| x_{i}\right\rangle}{\left\langle p_{f} \mid x_{i}\right\rangle}=\frac{1}{2} \int_{0}^{\infty} d T \int_{\tilde{x}(0)=0}^{\tilde{p}(T)=0} \mathcal{D} \tilde{x} \mathcal{D} \tilde{p} e^{-i \int_{0}^{T} d t\left(\tilde{p} \cdot p_{f}+\tilde{p} \cdot \dot{\tilde{x}}-Q_{0}^{B}(x(\tilde{x}), p(\tilde{p}))-i \epsilon\right)},
$$

where we have included the normalization factor $\left\langle p_{f} \mid x_{i}\right\rangle=e^{i p_{f} \cdot x_{i}}$. The need for such a factor is evident by considering the free case that should return $i\left(p_{f}^{2}-m^{2}\right)^{-1}$.

Before continuing with the calculation of eq. (2.9), we discuss a point that will become central for the spinning case in the next sections. Looking at eq. (2.9), one is tempted to pull $Q_{0}^{B}$ out of the integration over $t$, since it is a conserved charge. Of course, this is true only on the equations of motion, therefore the correct statement is formulated in terms of its expectation value, i.e.

$$
\int_{0}^{T} d t\left\langle Q_{0}^{B}(x(t), p(t))\right\rangle=\left\langle Q_{0}^{B}\left(x\left(t_{0}\right), p\left(t_{0}\right)\right)\right\rangle T,
$$

where $t_{0}$ is arbitrary. However, this is different from $\left\langle\left(Q_{0}^{B}\right)^{-1}\right\rangle$ which appears on the l.h.s. of eq. (2.9). Therefore, the only thing we could do on the same line of reasoning is to note that

$$
\frac{d}{d t}\left\langle\left(Q_{0}^{B}\right)^{-1}\right\rangle=-\left\langle\dot{Q}_{0}^{B}\left(Q_{0}^{B}\right)^{-2}\right\rangle .
$$

Then, a simple calculation from Noether's theorem reveals that a generic correlator of some operator $F\left[q_{i}\right]$ with the time derivative of a Noether charge $\dot{Q}\left[q_{i}\right]$ can be expressed in terms of the transformed canonical variables $\delta q_{i}(t) \operatorname{as}^{5}$

$$
\left\langle\dot{Q}\left[q_{i}\right] F\left[q_{i}\right]\right\rangle=-i \sum_{i}\left\langle\delta q_{i}(t) \frac{\delta F\left[q_{i}\right]}{\delta q_{i}(t)}\right\rangle .
$$

Therefore,

$$
\frac{d}{d t}\left\langle\left(Q_{0}^{B}\right)^{-1}\right\rangle=-i\left\langle\delta x_{\mu}(t) \frac{\delta\left(Q_{0}^{B}\right)^{-2}}{\delta x_{\mu}(t)}\right\rangle-i\left\langle\delta p_{\mu}(t) \frac{\delta\left(Q_{0}^{B}\right)^{-2}}{\delta p_{\mu}(t)}\right\rangle
$$

In the free case, the Noether charge $Q_{0}=\frac{1}{2}\left(p^{2}-m^{2}\right)$ does not depend on $x$ and the momentum is gauge invariant, i.e. $\delta p=0$. Hence, the r.h.s. of eq. (2.13) vanishes and one can effectively evaluate $Q_{0}(x(t), p(t))$ in the exponent of eq. (2.9) at an arbitrary time, say $T$. Then, the remaining integrations over $\tilde{p}^{\mu}$ and $\tilde{x}^{\mu}$ are trivial, and one gets the free Feynman propagator with momentum $p_{f}^{\mu}$.

However, for a generic dressed propagator, the charge $Q_{0}^{B}$ does depend on $x^{\mu}$ and the momentum $p^{\mu}$ is not gauge invariant. This makes eq. (2.13) more involved, so that it is actually more convenient to keep $Q_{0}^{B}$ inside the integral over $t$. To see how to proceed, we consider in the next section a specific case for the background field $B(x)$.

\footnotetext{
${ }^{5}$ We are assuming that the symmetry is non-anomalous.
} 


\subsection{Asymptotic propagators in a gauge boson background}

We consider a background gauge boson field $A^{\mu}$ that for simplicity we assume to be abelian. In this case, we define $Q_{0}^{A}$ from the quadratic part of the scalar QED Lagrangian as

$$
Q_{0}^{A}(\hat{x}, \hat{p}) \equiv \frac{1}{2}\left(\left(\hat{p}_{\mu}-A_{\mu}(\hat{x})\right)^{2}-m^{2}\right)=\frac{1}{2} \hat{p}^{2}-\hat{p} \cdot A(\hat{x})-i \frac{1}{2}(\partial \cdot A(\hat{x}))+\frac{1}{2} A^{2}(\hat{x}),
$$

where in the second equality we took into account that the path integral representation in eq. (2.7) requires a Weyl-ordered Hamiltonian.

Then, we can plug eq. (2.14) into eq. (2.9) and perform the Gaussian integration over $\tilde{p}$. We get

$$
\frac{\left\langle p_{f}\left|\left(2 Q_{0}^{A}+i \epsilon\right)^{-1}\right| x_{i}\right\rangle}{\left\langle p_{f} \mid x_{i}\right\rangle}=\frac{1}{2} \int_{0}^{\infty} d T e^{i \frac{1}{2}\left(p_{f}^{2}-m^{2}+i \epsilon\right) T} f\left(x_{i}, p_{f}, T\right),
$$

where we have defined

$$
\begin{aligned}
f\left(x_{i}, p_{f}, T\right)=\int_{\tilde{x}(0)=0} \mathcal{D} \tilde{x} \mathcal{P} \exp [ & i \int_{0}^{T} d t \frac{1}{2} \dot{\tilde{x}}^{2}+\left(p_{f}+\dot{\tilde{x}}\right) \cdot A\left(x_{i}+p_{f} t+\tilde{x}(t)\right) \\
& \left.+\frac{i}{2} \partial \cdot A\left(x_{i}+p_{f} t+x(t)\right)\right] .
\end{aligned}
$$

We see that eq. (2.15) represents a dressed propagator in terms of a radiative factor $f$, equal to unity in the free case, which takes into account the interactions with the background field via four one-dimensional fields $x^{\mu}(t)$ living on the worldline of proper time $T$.

Now we define the asymptotic dressed propagator as the dressed propagator truncated of the external free propagator of momentum $p_{f}$. This means that we should consider

$$
i\left(p_{f}^{2}-m^{2}\right) \frac{\left\langle p_{f}\left|\left(2 Q_{0}^{A}+i \epsilon\right)^{-1}\right| x_{i}\right\rangle}{\left\langle p_{f} \mid x_{i}\right\rangle}=\int_{0}^{\infty} d T\left(\frac{d}{d T} e^{i \frac{1}{2}\left(p_{f}^{2}-m^{2}+i \epsilon\right) T}\right) f\left(x_{i}, p_{f}, T\right) .
$$

Assuming that the dressed propagator in eq. (2.15) develops a simple pole for $p_{f}^{2} \rightarrow m^{2}$ with residue one and that the factor $f\left(x_{i}, p_{f}, T\right)$ remains finite in this limit, we can integrate by parts to get

$$
\lim _{p_{f}^{2} \rightarrow m^{2}} i\left(p_{f}^{2}-m^{2}\right) \frac{\left\langle p_{f}\left|\left(2 Q_{0}^{A}+i \epsilon\right)^{-1}\right| x_{i}\right\rangle}{\left\langle p_{f} \mid x_{i}\right\rangle}=\lim _{p_{f}^{2} \rightarrow m^{2}} f\left(x_{i}, p_{f}, \infty\right) .
$$

Therefore, the asymptotic dressed propagator equals eq. (2.16) in the limit $T \rightarrow \infty$. We are now ready to perform the remaining path integration in the soft expansion.

Following [28], we introduce a book-keeping parameter $\lambda$ and rescale $p_{f}^{\mu} \rightarrow \lambda n^{\mu}$, such that the soft expansion corresponds to an expansion in $1 / \lambda$. Accordingly, it is convenient to re-define the integration variable in eq. (2.16) as $t \rightarrow t / \lambda$. Then, we get

$$
f\left(x_{i}, n \lambda, \infty\right)=\int_{\tilde{x}(0)=0} \mathcal{D} \tilde{x} e^{i \int_{0}^{\infty} d t\left(\frac{\lambda}{2} \dot{\tilde{x}}^{2}+(n+\dot{\tilde{x}}) \cdot A\left(x_{i}+n t+\tilde{x}(t)\right)+\frac{i}{2 \lambda} \partial \cdot A\left(x_{i}+n t+\tilde{x}(t)\right)\right)} .
$$


The crucial observation is that all two-point correlators of $\tilde{x}^{\mu}$ and $\dot{\tilde{x}}^{\mu}$ are of order $1 / \lambda$ and thus at a given order in $1 / \lambda$ we need to include a finite number of diagrams. Therefore, by Taylor expanding $A_{\mu}$ in powers of $\tilde{x}^{\mu}$ we can solve this one-dimensional QFT order by order in $1 / \lambda$ and to all-orders in the coupling constant. More specifically, up to order $1 / \lambda^{m}$ we need the diagrams with at most $m$ propagators, hence with vertices with at most $2 m$ powers of $\tilde{x}^{\mu}$. Then, the sum of all diagrams can be rearranged in the exponential of the sum of connected diagrams. The calculation has been already carried out in detail in [28]. For the sake of completeness, here we review the most important steps, as these will be invoked in the derivation of the spinning cases in the following sections.

Let us start at leading power (LP) in $1 / \lambda$. In this case we set $\tilde{x}^{\mu}=0$, which means that we ignore the fluctuations around the classical straight path (see eq. (2.8)) and we evaluate the path integral on its stationary point. Thus eq. (2.19) becomes

$$
\begin{aligned}
f\left(x_{i}, n \lambda, \infty\right) & =\exp \left(i \int_{0}^{\infty} d t n^{\mu} A_{\mu}\left(x_{i}+n t\right)\right)+\mathcal{O}\left(\frac{1}{\lambda}\right) \\
& =\exp \left(-\int \frac{d^{d} k}{(2 \pi)^{d}} \frac{n^{\mu}}{n \cdot k} \tilde{A}_{\mu}(k) e^{i x_{i} \cdot k}\right)+\mathcal{O}\left(\frac{1}{\lambda}\right) .
\end{aligned}
$$

The term $e^{i x_{i} \cdot k}$ gives subleading corrections in the soft momentum $k$, so at LP we can safely set $x_{i}^{\mu}=0$. Therefore, at LP the asymptotic dressed propagator $f\left(x_{i}, n \lambda, \infty\right)$ reduces to the well-known straight Wilson line.

At NLP there are two sources of corrections: the first one comes from having $x_{i}^{\mu} \neq 0$ in eq. (2.20). This combines with the contribution from Low's theorem [28], to give the orbital angular momentum $L^{\mu \nu}$. Although this contribution does not exponentiate, the separation between the orbital and the spin contributions is not gauge invariant, as observed in [72]. Thus, it is convenient to put also $L^{\mu \nu}$ into the exponent, albeit regarding the expression as valid up to NNLP corrections.

The second source of corrections comes from including quantum fluctuations in the path integral. Up to order $1 / \lambda$ we need diagrams with only one propagator, which means that we need to expand the action up to second order in $\tilde{x}^{\mu}$ and $\dot{\tilde{x}}^{\mu}$. This yields

$$
\begin{aligned}
f(0, n \lambda, \infty)= & e^{-\frac{1}{2 \lambda} \int_{0}^{\infty} d t \partial \cdot A(n t)} \int_{\tilde{x}(0)=0} \mathcal{D} \tilde{x} \exp \left(i \int _ { 0 } ^ { \infty } d t \left(\frac{\lambda}{2} \dot{\tilde{x}}^{2}+\dot{\tilde{x}}^{\mu} A_{\mu}(n t)\right.\right. \\
& \left.\left.+\left(n^{\mu}+\dot{\tilde{x}}^{\mu}\right) \tilde{x}^{\nu} \partial_{\nu} A_{\mu}(n t)+n^{\mu} \tilde{x}^{\nu} \tilde{x}^{\rho} \partial_{\nu} \partial_{\rho} A_{\mu}(n t)\right)\right)+\mathcal{O}\left(\frac{1}{\lambda^{2}}\right) .
\end{aligned}
$$

The exponential with no power of $\tilde{x}^{\mu}$ yields

$$
\exp \left[\int \frac{d^{d} k}{(2 \pi)^{d}} \tilde{A}_{\mu}(k) \frac{k^{\mu}}{2 \lambda n \cdot k}\right] .
$$

Then, there are two class of connected diagrams with one propagator, as shown in figure 2: a loop-diagram with a single $\tilde{x}^{2}$ vertex, which yields

$$
-\int \frac{d^{d} k}{(2 \pi)^{d}} \tilde{A}_{\mu}(k) \frac{n^{\mu} k^{2}}{2 \lambda(n \cdot k)^{2}}
$$



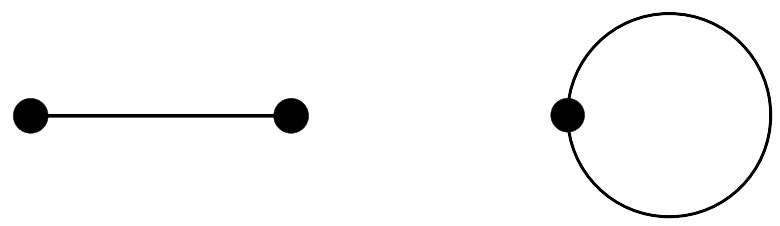

Figure 2. Connected diagrams needed up to order $1 / \lambda$ for the evaluation of the path integral in eq. (2.21). The diagram on the left corresponds to eq. (2.24), while the loop on the right corresponds to eq. $(2.23)$.

and a diagram where a propagator connects two $\tilde{x}^{1}$ vertices, which reads

$$
\begin{aligned}
\frac{1}{\lambda} \int \frac{d^{d} k}{(2 \pi)^{d}} \int \frac{d^{d} l}{(2 \pi)^{d}} \tilde{A}_{\mu}(k) \tilde{A}_{\nu}(l)\left(\frac{\eta^{\mu \nu}}{2 n \cdot(k+l)}-\frac{n^{\nu} l^{\mu} n \cdot k+n^{\mu} k^{\nu} n \cdot l}{2(n \cdot l)(n \cdot k)[n \cdot(k+l)]}\right. \\
\left.+\frac{(k \cdot l) n^{\mu} n^{\nu}}{2(n \cdot l)(n \cdot k)[n \cdot(k+l)]}\right) .
\end{aligned}
$$

It is noteworthy that eq. (2.24) contains two gauge fields, which means that NLP soft emissions at different times are correlated pairwise along the worldline. More generally, at $\mathrm{N}^{n} \mathrm{LP}$ we expect correlations among $n+1$ gauge bosons.

Exponentiating the sum of the connected diagrams in eq. (2.23) and eq. (2.24), and combining the result with eq. (2.20) and eq. (2.22), we conclude that the asymptotic dressed propagator $f\left(x_{i}, p_{f}, \infty\right)$ for a scalar particle reduces at NLP to the Generalized Wilson line defined in eq. (1.3) with $J^{\mu \nu}=L^{\mu \nu}$.

The derivation we have reviewed in this section can be generalized to the case of a nonabelian gauge field. In this case the dressed propagator becomes matrix-valued, hence we have two routes: either we introduce additional Grassmann variables on the worldline (as discussed in [24]) or we stick with matrices in the exponent after introducing a path ordering prescription. Although the former approach is more elegant and the quantization of the model is derived from first principles, the latter is often preferred in practical calculations involving soft gluons and it is the choice adopted in [28]. Therefore, also in this work we stick with the second option. Then, the definition of eq. (1.3) is essentially the same, although the exponentiation is not derived in terms of connected diagrams but the so-called webs $[28,31,32,113]$.

\section{Spin one-half}

Having reviewed the generalized Wilson line in the scalar case, we are going to present the Dirac case following the same procedure i.e. starting from the classical single-particle model and its symmetries. This will bring us to identify the distinctive features of asymptotic propagators and subsequently to justify eq. (1.3) for spin $1 / 2$.

\subsection{Worldline representation}

We start with the phase space action for a free massless spin $1 / 2$ particle, which reads

$$
S=\int d t\left(p_{\mu} \dot{x}^{\mu}+\frac{i}{2} \psi^{\mu} \dot{\psi}_{\mu}-H\right)
$$


where the Hamiltonian $H$ is

$$
H=\frac{1}{2} e p_{\mu} p^{\mu}+i \chi \psi_{\mu} p^{\mu}
$$

Here $\psi_{\mu}$ are classical Grassmann variables, which after quantization satisfy the Clifford algebra $\left\{\hat{\psi}_{\mu}, \hat{\psi}_{\nu}\right\}=\eta_{\mu \nu}$. Unlike the scalar case, this action enjoys two gauge symmetries: local supersymmetry and reparametrization invariance, with the respective gauge bosons $e(t)$ and $\chi(t)$. The transformations are

$$
\delta x^{\mu}=\xi p^{\mu}+i \zeta \psi^{\mu}, \quad \delta p^{\mu}=0, \quad \delta \psi^{\mu}=-\zeta p^{\mu}, \quad \delta e=\dot{\xi}, \quad \delta \chi=\dot{\zeta},
$$

while the relative Noether charges are

$$
Q_{0} \equiv \frac{1}{2} p^{2}, \quad Q_{1} \equiv \psi \cdot p,
$$

whose kernels define the physical Hilbert space. More specifically, the Dirac equation $Q_{1}|\psi\rangle=0$ generates the other constraint $Q_{0}|\psi\rangle=0$ thanks to the $N=1$ supersymmetry algebra $\left\{Q_{1}, Q_{1}\right\}=-2 i Q_{0}$.

Consequently, the Feynman propagator for the field $\psi(x)$ admits the following firstquantized representation

$$
\begin{aligned}
& \left\langle p_{f}\left|\frac{Q_{1}}{2 Q_{0}+i \epsilon}\right| x_{i}\right\rangle \\
& =\int \mathcal{D} e \mathcal{D} \chi \int_{\psi(1)=\Gamma} \mathcal{D} \psi \int_{x(0)=x_{i}}^{p(1)=p_{f}} \mathcal{D} x \mathcal{D} p e^{i p(1) \cdot x(1)-i \int_{0}^{1} d t\left(p \cdot \dot{x}+\frac{i}{2} \psi \cdot \dot{\psi}-e Q_{0}-\chi Q_{1}-i \epsilon\right)} .
\end{aligned}
$$

A few comments about the boundary conditions are in order. First, we note that as for the scalar case we are considering a mixed representation from an initial state of position $x_{i}^{\mu}$ (which eventually is integrated over) to a final state with momentum $p_{f}^{\mu}$. Then, we note that the propagator is open and thus we do not set antiperiodic boundary conditions $\psi_{\mu}^{\mu}(0)+\psi_{\mu}^{\mu}(1)=0$, as typically done for the computation of the effective action. In fact, it is known (see e.g. $[22,26]$ ) that an open propagator requires the inhomogeneous conditions $\psi_{\mu}^{\mu}(0)+\psi_{\mu}^{\mu}(1)=\Gamma^{\mu}$, where $\Gamma^{\mu}$ is a set of constant Grassmann variables that should generate the spin structure of the propagator. Given that the external states of a scattering amplitude must have a well-defined spin, while we sum over the spin values of the initial state attached to the hard function, we set only the final value of the spin variable by requiring $\psi_{\mu}(1)=\Gamma_{\mu}$, where $\Gamma_{\mu}$ will eventually be set proportional to the gamma matrices.

Then we can proceed as in the scalar case and gauge-fix the Lagrange multipliers, by setting $(e(t), \chi(t))=(T, \theta)$. The corresponding path integrations become regular integrals over the proper time $T$ and the "supertime" $\theta$, respectively. In this way, eq. (3.5) reads

$$
\begin{aligned}
\left\langle p_{f}\left|\frac{Q_{1}}{2 Q_{0}+i \epsilon}\right| x_{i}\right\rangle= & \frac{1}{2} \int_{0}^{\infty} d T \int d \theta \int_{\psi(T)=\Gamma} \mathcal{D} \psi \\
& \int_{x(0)=x_{i}}^{p(T)=p_{f}} \mathcal{D} x \mathcal{D} p e^{i p(T) \cdot x(T)-i \int_{0}^{T} d t\left(p \cdot \dot{x}+\frac{i}{2} \psi \cdot \dot{\psi}-Q_{0}-\frac{\theta}{T} Q_{1}-i \epsilon\right) .}
\end{aligned}
$$


Once again, the role of the Lagrange multipliers is to exponentiate the constraints $Q_{0}$ and $Q_{1}$. In particular, the Grassmann nature of the supertime $\theta$ allows the exponentiation of the numerator of the propagator, while the proper time $T$ exponentiates the denominator as for the scalar case.

The generalization to the presence of an abelian gauge boson background field is straightforward. This can be achieved by replacing $p_{\mu} \rightarrow p_{\mu}-A_{\mu} \equiv \Pi_{\mu}$ in the charge $Q_{1}$, while the charge $Q_{0}$ is obtained by the supersymmetry algebra $\left\{Q_{1}, Q_{1}\right\}=-2 i Q_{0}$. The new transformations read

$$
\begin{aligned}
& \delta x^{\mu}=\xi \Pi \mu+i \zeta \psi^{\mu}, \quad \delta p^{\mu}=-\frac{\xi}{2} \frac{\delta \Pi^{2}}{\delta x_{\mu}}-i \zeta \psi_{\nu} \frac{\delta \Pi^{\nu}}{\delta x_{\mu}}, \quad \delta \psi^{\mu}=-\zeta \Pi^{\mu}, \\
& \delta e=\dot{\xi}+2 i \chi \zeta, \quad \delta \chi=\dot{\zeta},
\end{aligned}
$$

and are generated by the new charges

$$
Q_{0}^{A} \equiv \Pi^{2}+\psi_{\mu} \psi_{\nu} F^{\mu \nu}, \quad Q_{1}^{A} \equiv \psi \cdot \Pi
$$

Then, the structure of eq. (3.6) remains the same.

At this point, we note that unlike the scalar case where the dressed propagator is represented by the expectation values of the inverse of a conserved charge, here we have an additional charge in the numerator. Therefore, we can directly apply eq. (2.10) and evaluate $Q_{1}^{A}$ in the exponent of eq. (3.6) at an arbitrary time and pull it out of the integral. Given the boundary conditions that fix $\psi(T)$, we choose this arbitrary time to be $T$. Therefore we consider

$$
\int_{0}^{T} d t\left\langle Q_{1}^{A}(x(t), p(t))\right\rangle=\left\langle Q_{1}^{A}\left(x(T), p_{f}\right)\right\rangle T
$$

and eq. (3.6) becomes

$$
\begin{aligned}
\left\langle p_{f}\left|\frac{Q_{1}^{A}}{2 Q_{0}^{A}+i \epsilon}\right| x_{i}\right\rangle= & \frac{1}{2} \int_{0}^{\infty} d T \int d \theta \int_{\psi(T)=\Gamma} \mathcal{D} \psi \\
& \int_{x(0)=x_{i}}^{p(T)=p_{f}} \mathcal{D} x \mathcal{D} p e^{i p(T) \cdot x(T)+i \theta Q_{1}^{A}\left(x(T), p_{f}\right)-i \int_{0}^{T} d t\left(p \cdot \dot{x}+\frac{i}{2} \psi \cdot \dot{\psi}-Q_{0}^{A}-i \epsilon\right)} .
\end{aligned}
$$

This is the worldline representation for a Dirac propagator in the presence of an abelian background field with boundary conditions suitably chosen to handle the asymptotic states of a scattering amplitudes. In the following sections we are going to discuss how such representation is related to the numerator and the denominator of a dressed propagator, respectively.

\subsection{Numerator contribution}

The presence of $x(T)$ in the argument of the numerator contribution $Q_{1}^{A}$ in eq. (3.10) seems to suggest that the procedure outlined above is pointless, since $x(T)$ is not fixed by the boundary conditions. However, there are at least two cases where this can be handled: the free case and, most importantly, the asymptotic case. We start with the former. 
In the free case the gauge field vanishes so the Noether charges $Q_{0}$ and $Q_{1}$ do not depend on $x_{\mu}$. Making use of the boundary conditions on $p_{\mu}$ and $\psi_{\mu}$ we get

$$
Q_{1}(x(T), p(T))=\Gamma \cdot p_{f}
$$

This gives

$$
\begin{aligned}
\left\langle p_{f}\left|\frac{Q_{1}}{2 Q_{0}+i \epsilon}\right| x_{i}\right\rangle=e^{i p_{f} \cdot x_{i}} & \frac{1}{2} \int_{0}^{\infty} d T e^{\frac{i}{2}\left(p_{f}^{2}+i \epsilon\right) T} \int d \theta e^{i \theta \Gamma \cdot p_{f}} \\
& \left.\int_{\psi(T)=\Gamma} \mathcal{D} \psi e^{\int_{0}^{T} d t \frac{1}{2} \psi \cdot \dot{\psi}} \int_{\tilde{x}(0)=0}^{\tilde{p}(T)=0} \mathcal{D} \tilde{x} \mathcal{D} \tilde{p} e^{i \int_{0}^{T} d t\left(\frac{\tilde{p}^{2}}{2}+\tilde{p} \cdot \dot{\tilde{x}}\right.}\right) .
\end{aligned}
$$

The remaining path integrals are Gaussian and can be absorbed together with $e^{i p_{f} \cdot x_{i}}$ into the normalization factor $\left\langle p_{f} \mid x_{i}\right\rangle$. Then, assuming that the constant $\Gamma_{\mu}$ can be represented by the Dirac gamma matrices $\gamma_{\mu}$, one is left with

$$
\left\langle p_{f} \mid x_{i}\right\rangle^{-1}\left\langle p_{f}\left|\frac{Q_{1}}{2 Q_{0}+i \epsilon}\right| x_{i}\right\rangle=\frac{1}{2} \int_{0}^{\infty} d T e^{\frac{i}{2}\left(p_{f}^{2}+i \epsilon\right) T} \int d \theta e^{i p_{f} \theta}=\frac{i \not p_{f}}{p_{f}^{2}+i \epsilon},
$$

as expected.

Coming back to the interacting case in eq. (3.10), we first note that the Noether charge with the given boundary conditions reads

$$
Q_{1}^{A}(x(T), p(T))=\Gamma \cdot\left(p_{f}-A(x(T))\right) .
$$

Then, it is convenient to get rid of the Gaussian integration over $p_{\mu}$, to get

$$
\left\langle p_{f} \mid x_{i}\right\rangle^{-1}\left\langle p_{f}\left|\frac{Q_{1}^{A}}{2 Q_{0}^{A}+i \epsilon}\right| x_{i}\right\rangle=\frac{1}{2} \int_{0}^{\infty} d T e^{\frac{i}{2}\left(p_{f}^{2}+i \epsilon\right) T} \int d \theta e^{i \Gamma \cdot p_{f} \theta} f\left(x_{i}, p_{f}, \Gamma, T, \theta\right),
$$

where, in analogy with eq. (2.16), we have defined

$$
\begin{aligned}
& f\left(x_{i}, p_{f}, \Gamma, T, \theta\right)=\int_{\psi(T)=\Gamma} \mathcal{D} \psi \int_{\tilde{x}(0)=0} \mathcal{D} \tilde{x} e^{-i \theta \psi \cdot A\left(x_{i}+p_{f} T+\tilde{x}(T)\right)} \\
& \exp \left(i \int _ { 0 } ^ { T } d t \left(-\frac{i}{2} \psi \cdot \dot{\psi}+\frac{1}{2} \dot{\tilde{x}}^{2}+\left(p_{f}+\dot{\tilde{x}}\right) \cdot A\left(x_{i}+p_{f} t+\tilde{x}(t)\right)\right.\right. \\
&\left.\left.\quad+\frac{i}{2} \partial \cdot A\left(x_{i}+p_{f} t+\tilde{x}(t)\right)+\frac{1}{2} \psi_{\mu} \psi_{\nu} F^{\mu \nu}\right)\right)
\end{aligned}
$$

Now, for asymptotic propagators we should truncate the external line by multiplying by a free inverse propagator, similarly to eq. (2.17). Hence, we should consider

$$
\begin{aligned}
\bar{u}\left(p_{f}\right) \frac{i}{p_{f f}} p_{f}^{2}\left\langle p_{f} \mid x_{i}\right\rangle^{-1} & \left\langle p_{f}\left|\frac{Q_{1}^{A}}{2 Q_{0}^{A}+i \epsilon}\right| x_{i}\right\rangle \\
& =\bar{u}\left(p_{f}\right) \int_{0}^{\infty} d T \frac{d}{d T}\left(e^{\frac{i}{2}\left(p_{f}^{2}+i \epsilon\right) T}\right) \int d \theta e^{i \Gamma \cdot p_{f} \theta} f\left(x_{i}, p_{f}, \Gamma, T, \theta\right) .
\end{aligned}
$$


With similar assumptions leading to eq. (2.18), we can integrate eq. (3.17) by parts to get

$$
\bar{u}\left(p_{f}\right) \frac{i}{p / f} p_{f}^{2}\left\langle p_{f} \mid x_{i}\right\rangle^{-1}\left\langle p_{f}\left|\frac{Q_{1}^{A}}{2 Q_{0}^{A}+i \epsilon}\right| x_{i}\right\rangle=\lim _{p_{f}^{2} \rightarrow 0} \bar{u}\left(p_{f}\right) \frac{1}{p / f} \int d \theta e^{i \Gamma \cdot p_{f} \theta} f\left(x_{i}, p_{f}, \Gamma, \infty, \theta\right) .
$$

Therefore, also in the Dirac case, the asymptotic propagator is obtained by taking the $T \rightarrow \infty$ limit of the dressed propagator, in this case of eq. (3.16).

The practical consequence is that the gauge field $A_{\mu}(x(T))$ in eq. (3.14) is evaluated at infinity. Let us assume for a moment that it vanishes. Then, we can drop the $\theta$-dependence in $f\left(x_{i}, p_{f}, \Gamma, \infty, \theta\right)$ and the $\theta$-integral in eq. (3.18) cancels with $\not p_{f}$ in the denominator. Therefore, the asymptotic dressed propagator in the presence of an asymptotically vanishing background field reads

$$
\lim _{p_{f}^{2} \rightarrow 0} \bar{u}\left(p_{f}\right) f\left(x_{i}, p_{f}, \Gamma, \infty\right)
$$

In other words, the numerator $Q_{1}^{A}$ of the dressed propagator has been taken effectively free, leaving the entire dependence on the background field in the denominator $Q_{0}^{A}$. This is very reminiscent of the closed loop topology of the one-loop effective action, as pointed out in the introduction.

At this point we should examine more carefully the assumption of the vanishing $A_{\mu}(x(T))$. In fact, what we should consider is the asymptotic limit in the soft expansion. We have seen in the scalar case that such expansion is achieved by rescaling $p_{f}^{\mu} \rightarrow \lambda n^{\mu}$ and then expanding in $1 / \lambda$. Looking at eq. (3.14) we can immediately see that $A_{\mu}(x(T))$ is subleading w.r.t. $p_{f}^{\mu}$. This is reassuring, since it is well-known that one should get a regular Wilson line in the strict soft limit, which is insensitive to the spin of the emitter. The question is whether $A_{\mu}(x(T))$ can also be neglected at subleading power in the soft expansion.

The answer is yes. This can be seen by mimicking what done in section 2 for the scalar case: after expanding the gauge field in powers of $\tilde{x}^{\mu}$, the only relevant vertex up to order $1 / \lambda$ is

$$
\Gamma \cdot A\left(p_{f} T\right)=\Gamma^{\mu} \int \frac{d^{d} k}{(2 \pi)^{d}} e^{i k \cdot p_{f} T} \tilde{A}_{\mu}(k) .
$$

This contains no power of $\tilde{x}^{\mu}$ and can thus be pulled out of the path integral. Vertices with higher powers of $\tilde{x}^{\mu}$ would be needed in diagrams with $\tilde{x}$-propagators, which are thus subleading in $1 / \lambda$.

The integral in eq. (3.20) is suppressed if the integrand oscillates very rapidly, as happens in the asymptotic limit $T \rightarrow \infty$, assuming an integrable $\tilde{A}_{\mu}(k)$. An obvious counter-example is given by the limiting case of a constant field, where the integrand has support only at $k=0$. One might wonder whether the soft limit is dangerous here since it corresponds to a long-wavelength background field with $\tilde{A}_{\mu}(k)$ concentrated around $k \rightarrow 0$. However, the soft limit on the worldline has been defined by rescaling $p_{f} \rightarrow \lambda n$ and letting $\lambda \rightarrow \infty$ so that $p_{f} \cdot k$ does not tend to zero. In other words, while it is legitimate to assume a constant field strength tensor $F^{\mu \nu}$ in this limit, the field $A^{\mu}$ is not exactly constant and it 
vanishes for $T \rightarrow \infty$. Therefore, eq. (3.20) vanishes and we conclude that for an asymptotic propagator dressed by (next-to-)soft radiation the numerator contribution $Q_{1}^{A}$ reduces to the free numerator $Q_{1}$.

\subsection{Denominator contribution}

Now that we have established that eq. (3.19) holds for asymptotic propagators dressed of soft radiation, we can perform the remaining path integrations in eq. (3.16) in the limit $T \rightarrow$ $\infty$. In principle we could integrate out $\psi$ exactly, since it appears quadratically. However, this will bring an intricate dependence on the background field. ${ }^{6}$ For our purposes it is actually more convenient to perform the integration order by order in the soft expansion, where we can use the same rescaling implemented in the $x$-integration, i.e. $p_{f} \rightarrow \lambda n$ and $t \rightarrow t / \lambda$, to get

$$
\int_{\psi(\infty)=\Gamma} \mathcal{D} \psi e^{i \int_{0}^{\infty} d t\left(-\frac{i}{2} \psi \cdot \dot{\psi}+\frac{1}{2 \lambda} \psi_{\mu} \psi_{\nu} F^{\mu \nu}\right)}
$$

It is convenient to expand the field $\psi_{\mu}(t)$ around the boundary condition with the replacement $\psi(t)=\psi(T)+\widetilde{\psi}(t)$. In this way eq. (3.21) becomes

$$
e^{\frac{i}{2 \lambda} \Gamma_{\mu} \Gamma_{\nu} \int_{0}^{\infty} d t F^{\mu \nu}\left(x_{i}+n t+\tilde{x}(t)\right)} \int_{\widetilde{\psi}(\infty)=0} \mathcal{D} \widetilde{\psi} e^{i \int_{0}^{\infty} d t\left(-\frac{i}{2} \widetilde{\psi} \cdot \tilde{\psi}+\frac{1}{2 \lambda} \widetilde{\psi}_{\mu} \widetilde{\psi}_{\nu} F^{\mu \nu}\left(x_{i}+n t+\tilde{x}(t)\right)\right)} .
$$

We note that the propagator $\left\langle\widetilde{\psi}(t) \widetilde{\psi}\left(t^{\prime}\right)\right\rangle$ is of order $\lambda^{0}$ and is proportional to the step function $\theta\left(t-t^{\prime}\right)$. The interaction term is of order $\lambda^{-1}$ and generates vertices with various powers of $\tilde{x}$ by expanding $A_{\mu}(x(t))=A_{\mu}\left(x_{i}+n t+\tilde{x}(t)\right)$ around $\tilde{x}=0$. As already observed in the scalar case, the effect from $x_{i} \neq 0$ is subleading in $1 / \lambda$. Moreover, here this is multiplied by an additional $1 / \lambda$ coming from the vertex, hence it is a $1 / \lambda^{2}$ effect that we can neglect. Now we recall that the propagator of the $\tilde{x}(t)$ field (and more generally all correlators with two powers of $\tilde{x}$ or $\dot{\tilde{x}}$ ) are of order $1 / \lambda$. Therefore, vertices with higher powers of $\tilde{x}$ are needed only for diagrams that are subleading in the soft expansion. Thus we can expand the gauge field in $F_{\mu \nu}$ at leading order in $\tilde{x}$ so that no dependence over $\tilde{x}$ is left and the path integral over $\widetilde{\psi}$ in eq. (3.16) decouples from the one over $\tilde{x}$.

In analogy with the free case of eq. (3.13), we can absorb such remaining Gaussian integration over $\widetilde{\psi}$ in the normalization factor $\left\langle p_{f} \mid x_{i}\right\rangle$, so that one is left with the factor

$$
\exp \left(\frac{i}{2 \lambda} \Gamma_{\mu} \Gamma_{\nu} \int_{0}^{\infty} d t F^{\mu \nu}(n t)\right)=\exp \left(\frac{i}{\lambda} S^{\mu \nu} \int \frac{d^{d} k}{(2 \pi)^{d}} \frac{k_{\nu}}{n \cdot k} \tilde{A}_{\mu}(k)\right)
$$

where we used the representation $\Gamma_{\mu}=\frac{1}{\sqrt{2}} \gamma_{\mu}$ and we introduced the spin $1 / 2$ generator $S^{\mu \nu}=\frac{i}{4}\left[\gamma^{\mu}, \gamma^{\nu}\right]$. Also, we set $x_{i}=0$ since it is a subleading effect, as discussed in section 2 . As we can see, eq. (3.23) equals the fourth term in the first line of eq. (1.3).

The remaining path integral over $\tilde{x}$ matches exactly the scalar case and therefore we conclude that the only difference between the Dirac and the scalar case is given by the result in eq. (3.23), in agreement with [28]. As we have remarked in the introduction

\footnotetext{
${ }^{6}$ The result significantly simplifies for a constant background field [21].
} 
with eq. (1.4), it is a pure "denominator" effect, which is present also in the effective action [12]. It represents a chromo-magnetic interaction between the emitter and the nextto-soft radiated particles by the coupling of $F^{\mu \nu}$ with the Lorentz generator $S^{\mu \nu}$. This is precisely the spin term in the generalized Wilson line of eq. (1.3).

So far we assumed an abelian background field. A similar derivation can be presented in the non-abelian case, by assuming that the exponentials are path-ordered. One still ends up with eq. (3.21). However, in this case $F^{\mu \nu}$ contains the commutator $\left[A^{\mu}, A^{\nu}\right]$ which gives rise to the following spin-dependent term

$$
\frac{i g^{2}}{2 \lambda} \int_{0}^{\infty} d t \Gamma^{\mu} \Gamma^{\nu}\left[A_{\mu}(n t), A_{\nu}(n t)\right]=\frac{i g^{2}}{\lambda} S^{\mu \nu} \int \frac{d^{d} k}{(2 \pi)^{d}} \int \frac{d^{d} l}{(2 \pi)^{d}} \frac{1}{n \cdot(k+l)} \tilde{A}_{\mu}(k) \tilde{A}_{\nu}(l),
$$

where we exploited the anti-symmetry of the Lorentz generator. As we can see, eq. (3.24) reproduces the spin-dependent term with two gauge fields in eq. (1.3).

We conclude this section with a small remark about the introduction of a mass term, which is notoriously subtle for Dirac particles. Traditionally, this requires an additional spin variable $\psi_{5}[6,17,20,26,114]$. This is a necessary choice if one wants to incorporate the mass term in the extended Hamiltonian of eq. (3.2), since the additional variable would make the term Grassmann-even. Alternatively, it is sufficient to leave it out of the path integral as a Grassmann-odd projector, as already proposed in [16, 22]. However, for the purpose of this paper one is forced to include this extra dimensional variable in order to justify the vanishing contribution of the gauge field in the numerator for asymptotic propagators. This can be achieved with the conserved charges

$$
Q_{0}^{A} \equiv \Pi^{2}+\psi_{\mu} \psi_{\nu} F^{\mu \nu}-m^{2}, \quad Q_{1}^{A} \equiv \psi \cdot \Pi+m \psi_{5}
$$

The derivation then is analogous: both the mass term in the numerator and in the denominator can be factored out and cancel with the free inverse propagator.

\section{Spin one}

\subsection{Generalized Wilson Line for gluons}

Before discussing the details of the supersymmetric worldline model for higher spin particles, it is instructive to derive the GWL for gluons starting from the dressed propagator of the corresponding field-theory, rather than the quantization of the relativistic particle. In fact, as we have seen in the Dirac case, the model with worldline fermions is introduced in this context to explain the role of the background field in the numerator of dressed propagators. However, for spin-one we can use gauge invariance as a shortcut. Indeed, recalling that the GWLs are meant to represent the external states of a scattering amplitude, the dressed propagator can be computed in a gauge where the numerator is unity and thus does not depend on the background gauge field. Let us discuss this in more detail.

We start from the quadratic part of the field-theory Lagrangian. In order to preserve the gauge invariance w.r.t. the (soft) background field, it is convenient to work in the class of background-field-gauges. This is a well-known procedure (see e.g. [115]), which consists 
of replacing $A^{\mu} \rightarrow \widetilde{A}^{\mu}+A^{\mu}$ in the Yang-Mills Lagrangian $\mathcal{L}=-\frac{1}{4}\left(F_{\mu \nu}^{a}\right)^{2}$, and subsequently fix the gauge by adding the term

$$
\mathcal{L}_{\text {gf }}=-\frac{1}{2 \xi}\left(\widetilde{D}_{\mu} A^{\mu}\right)^{2}
$$

with the corresponding ghost term

$$
\mathcal{L}_{\text {ghost }}=\left(\widetilde{D}_{\mu} \bar{\omega}\right)^{a}\left(\widetilde{D}_{\mu} \omega\right)^{a}+g f^{a b c}\left(\widetilde{D}_{\mu} \bar{\omega}\right)^{a} A_{\mu}^{b} \omega^{c} .
$$

Here, we defined

$$
\widetilde{D}_{\mu}^{a b}=\partial_{\mu} \delta^{a b}-g f^{a b c} \widetilde{A}_{\mu}^{c},
$$

where we chose $\widetilde{A}^{\mu}$ to be the background field. Importantly, this gauge-fixing term breaks the invariance only w.r.t. the propagating $A^{\mu}$ field, while the gauge symmetry of $\widetilde{A}^{\mu}$ is preserved. Then, after some algebra, the quadratic part reads

$$
\mathcal{L}_{A^{2}}=\frac{1}{2} A_{\mu}^{a}\left[\eta^{\mu \nu}\left(\widetilde{D}^{a b}\right)^{2}-\left(1-\frac{1}{\xi}\right)\left(\widetilde{D}^{\mu} \widetilde{D}^{\nu}\right)^{a b}+i g f^{a b c} \widetilde{F}_{\rho \sigma}^{c}\left(S^{\mu \nu}\right)^{\rho \sigma}\right] A_{\nu}^{b}
$$

where we introduced the spin-one Lorentz generator

$$
\left(S_{\mu \nu}\right)^{\rho \sigma}=i\left(\delta_{\mu}^{\rho} \delta_{\nu}^{\sigma}-\delta_{\nu}^{\rho} \delta_{\mu}^{\sigma}\right) .
$$

Therefore, setting $\xi=1$ and taking the inverse of the expression in brackets in eq. (4.4), we see that the numerator of the dressed propagator is unity. The denominator on the other hand has the same structure that we found in the spinor case, i.e. a scalar term $D^{2}$ and a spin-dependent term $F_{\mu \nu} S^{\mu \nu}$ representing a chromo-magnetic interaction between the magnetic moment of the emitting particle and the background field. This leads us to define the following Hamiltonian

$$
H^{\mu \nu, a b}=\frac{1}{2}\left(\eta^{\mu \nu}\left(D^{a b}\right)^{2}+i g f^{a b c} F_{\rho \sigma}^{c}\left(S^{\mu \nu}\right)^{\rho \sigma}\right),
$$

where we have dropped the tilde over the background field. Thus, by replacing $\partial_{\mu} \rightarrow-i \hat{p}_{\mu}$ the covariant derivative can be written as an operator in the Hilbert space generated by $\hat{x}$ and $\hat{p}$, i.e.

$$
D_{\mu}^{a b}(\hat{x}, \hat{p})=-i \hat{p}_{\mu} \delta^{a b}+i g A_{\mu}^{a b}(\hat{x}),
$$

where we defined as usual

$$
A_{\mu}^{a b}=A_{\mu}^{c} T_{c}^{a b}=-i f^{a b c} A_{\mu}^{c}, \quad F_{\mu \nu}^{a b}=F_{\mu \nu}^{c} T_{c}^{a b}=-i f^{a b c} F_{\mu \nu}^{c} .
$$

Then, we can proceed as in the scalar case and consider the following path integral representation for the dressed propagator with a mixed position-momentum boundary conditions:

$$
\begin{aligned}
\left\langle p_{f}\left|\left(H_{\mu \nu}+i \epsilon\right)^{-1}\right| x_{i}\right\rangle & =\frac{1}{2} \int_{0}^{\infty} d T\left\langle p_{f}\left|e^{-i\left(H_{\mu \nu}(\hat{x}, \hat{p})+i \epsilon\right) T}\right| x_{i}\right\rangle \\
& =\frac{1}{2} \int_{0}^{\infty} d T \int_{x(0)=x_{i}}^{p(T)=p_{f}} \mathcal{D} x \mathcal{D} p \mathcal{P} e^{i p(T) \cdot x(T) \eta_{\mu \nu}-i \int_{0}^{T} d t\left(p \cdot \dot{x} \eta_{\mu \nu}-H_{\mu \nu}(\hat{x}, \hat{p})-i \epsilon\right)}
\end{aligned}
$$


where for notation purposes color indices have not been explicitly shown. Performing the Gaussian integration over the momentum and factorizing the normalization factor $\left\langle p_{f} \mid x_{i}\right\rangle$, we get

$$
\frac{\left\langle p_{f}\left|\left(H_{\mu \nu}+i \epsilon\right)^{-1}\right| x_{i}\right\rangle}{\left\langle p_{f} \mid x_{i}\right\rangle}=\frac{1}{2} \int_{0}^{\infty} d T e^{i \frac{1}{2}\left(p_{f}^{2}+i \epsilon\right) T} f_{\mu \nu}\left(x_{i}, p_{f}, T\right) .
$$

This expression can be compared with the corresponding representations in eq. (2.15) and eq. (3.15). Here, in analogy with eq. (2.16) and eq. (3.16), we defined

$$
\begin{aligned}
f_{\mu \nu}\left(x_{i}, p_{f}, T\right)= & \int_{\tilde{x}(0)=0} \mathcal{D} \tilde{x} \mathcal{P} \exp \left[i \int _ { 0 } ^ { T } d t \left(\frac{1}{2} \dot{\tilde{x}}^{2}+\left(p_{f}+\dot{\tilde{x}}\right) \cdot A\left(x_{i}+p_{f} t+\tilde{x}(t)\right)\right.\right. \\
& \left.\left.+\frac{i}{2} \partial \cdot A\left(x_{i}+p_{f} t+\tilde{x}(t)\right)\right) \eta_{\mu \nu}+g\left(S_{\mu \nu}\right)^{\rho \sigma} F_{\rho \sigma}\left(x_{i}+p_{f} t+\tilde{x}(t)\right)\right],
\end{aligned}
$$

where once again we have expanded around the classical solutions given by eq. (2.8).

Since the numerator of the dressed propagator is proportional to $\eta_{\mu \nu}$, the truncation of the external propagator is harmless. Indeed, we get

$$
\epsilon_{\mu}^{*}\left(p_{f}\right)\left(i \eta^{\mu \rho} p_{f}^{2}\right) \frac{\left\langle p_{f}\left|\left(H_{\rho \nu}+i \epsilon\right)^{-1}\right| x_{i}\right\rangle}{\left\langle p_{f} \mid x_{i}\right\rangle}=\epsilon^{* \mu}\left(p_{f}\right) \int_{0}^{\infty} d T\left(\frac{d}{d T} e^{i \frac{1}{2}\left(p_{f}^{2}+i \epsilon\right) T}\right) f_{\mu \nu}\left(x_{i}, p_{f}, T\right) .
$$

Integrating by parts and taking the on-shell limit $p_{f}^{2} \rightarrow 0$, eq. (4.12) reduces to

$$
\lim _{p_{f}^{2} \rightarrow 0} \epsilon^{* \mu}\left(p_{f}\right) f_{\mu \nu}\left(x_{i}, p_{f}, \infty\right) .
$$

Therefore, in analogy with the scalar and the Dirac cases, the dressed asymptotic propagator for a gluon is given by eq. (4.11) in the limit $T \rightarrow \infty$.

Finally, the path integral can be solved order by order in the soft expansion after replacing $p_{f}^{\mu} \rightarrow \lambda n^{\mu}$ and considering only diagrams up to order $1 / \lambda$, as discussed in section 2 for the scalar case. Here, the only difference is given by the presence of the $F_{\mu \nu}\left(S_{\mu \nu}\right)^{\rho \sigma}$ term. By expanding the gauge field in $F_{\mu \nu}$ at leading order in $\tilde{x}^{\mu}$ we get two additional terms of order $1 / \lambda$. The first involves one power of $A^{\mu}$ :

$$
\frac{i g}{\lambda} \int_{0}^{\infty} d t\left(S_{\mu \nu}\right)^{\rho \sigma} \partial_{\rho} A_{\sigma}(n t)=\frac{i g}{\lambda}\left(S_{\mu \nu}\right)^{\rho \sigma} \int \frac{d^{d} k}{(2 \pi)^{d}} \frac{k_{\sigma}}{n \cdot k} \tilde{A}_{\rho}(k) .
$$

The second one contains the non-abelian term $\left[A_{\mu}, A_{\nu}\right]$. Exploiting the anti-symmetry of $\left(S_{\mu \nu}\right)^{\rho \sigma}$ yields

$$
\frac{g^{2}}{\lambda} \int_{0}^{\infty} d t\left(S_{\mu \nu}\right)^{\rho \sigma} A_{\rho}(n t) A_{\sigma}(n t)=\frac{g^{2}}{\lambda}\left(S_{\mu \nu}\right)^{\rho \sigma} \int \frac{d^{d} k}{(2 \pi)^{d}} \int \frac{d^{d} l}{(2 \pi)^{d}} \frac{1}{n \cdot(k+l)} \tilde{A}_{\rho}(k) \tilde{A}_{\sigma}(l) .
$$

Neither term contains a power of $\tilde{x}^{\mu}$. Hence, the path integral can be performed precisely as in the scalar case, thus showing that soft gluon emissions naturally exponentiate at NLP also for spin-one emitters. Finally, including the angular momomentum generator $L_{\mu \nu}$ into the exponent for gauge transformation purposes (as remarked in footnote 2) leads to the generalized Wilson line defined in eq. (1.3). 


\subsection{The supersymmetric model}

At this point we have achieved our goal, since we have shown that eq. (1.3) is a suitable representation for the asymptotic states of a scattering amplitude also in the spin-one case. However, it would be desirable to explore the connection of eq. (1.3) with the supersymmetric worldline model, for several reasons. The first one is that this analysis would make a better parallel with the method presented in section 2 and section 3. More importantly, one would like to investigate whether there exists a wordline representation that is suitable for a propagator whose numerator is not unity, such as for massive vector bosons or gluons in generic gauges, and more generally for particles of higher spin. A complete and detailed solution to this problem is beyond the scope of this paper. Here, we limit our analysis to the $N=2$ model in four dimensions, ${ }^{7}$ following the same strategy adopted in section 3 and highlighting the typical features and difficulties that one encounters in the study of the asymptotic dynamics for spin higher than $1 / 2$.

We consider eq. (1.2) for $N=2$. Following [9] and [116], we first redefine our variables via

$$
\begin{aligned}
\psi^{\mu} & =\frac{1}{\sqrt{2}}\left(\psi_{1}^{\mu}+i \psi_{2}^{\mu}\right), & \bar{\psi}^{\mu} & =\frac{1}{\sqrt{2}}\left(\psi_{1}^{\mu}-i \psi_{2}^{\mu}\right), \\
\chi^{\mu} & =\frac{1}{\sqrt{2}}\left(\chi_{1}^{\mu}+i \chi_{2}^{\mu}\right), & \bar{\chi}^{\mu} & =\frac{1}{\sqrt{2}}\left(\chi_{1}^{\mu}-i \chi_{2}^{\mu}\right) .
\end{aligned}
$$

Then, the action reads

$$
S=\int d t\left(p_{\mu} \dot{x}^{\mu}+i \bar{\psi}^{\mu} \dot{\psi}_{\mu}-H\right)
$$

where the Hamiltonian $H$ is

$$
H=\frac{1}{2} e p_{\mu} p^{\mu}+i \bar{\chi} \psi_{\mu} p^{\mu}+i \chi \bar{\psi}_{\mu} p^{\mu}-a \bar{\psi}_{\mu} \psi^{\mu} .
$$

The tranformations for reparametrization invariance, $N=2$ local supersymmetry and $O(2)$ symmetry are respectively generated by

$$
Q_{0} \equiv \frac{1}{2} p^{2}, \quad Q_{1} \equiv \psi \cdot p, \quad Q_{2} \equiv \bar{\psi} \cdot p, \quad J \equiv \bar{\psi} \cdot \psi .
$$

The $O(N)$ symmetry, with gauge field $a$, is a distinctive feature of particles with spin $N \geq 1$.

The quantization of this model on the closed line topology has been carried in detail in [116-118] while the free open propagator has been discussed in [18]. Here, in analogy with section 2 and section 3, we must consider a path integral representation for the open line compatible with the less common boundary conditions of asymptotic dressed propagators, i.e.

$$
\begin{aligned}
\int \mathcal{D} e \mathcal{D} \chi \mathcal{D} \bar{\chi} \mathcal{D} a \int_{\psi(1)=\Gamma}^{\bar{\psi}(1)=\bar{\Gamma}} \mathcal{D} \psi \mathcal{D} \bar{\psi} \\
\int_{x(0)=x_{i}}^{p(1)=p_{f}} \mathcal{D} x \mathcal{D} p e^{i p(1) \cdot x(1)-i \int_{0}^{1} d t\left(p \cdot \dot{x}+i \bar{\psi} \cdot \dot{\psi}-e Q_{0}-\bar{\chi} Q_{1}-\chi Q_{2}+a J-i \epsilon\right) .}
\end{aligned}
$$

\footnotetext{
${ }^{7}$ The quantization in $d$-dimension is more subtle (see e.g. [116]).
} 
Now we fix the gauge multiplet. The einbein $e$ can be set equal to the proper time $T$, as usual. Unlike the close topology of [116], the Grassmann variables $\chi$ and $\bar{\chi}$ cannot be set to zero, since they must generate the spin structure of the propagator, in analogy with the Dirac case of section 3. The gauge field $a$ deserves special attention, since its role is to set the degrees of freedom that one wish to propagate on the worldline. From this point of view, the choice $a=0$ is not the best one, since the corresponding propagator carries undesired remainder terms [18]. However, it provides a great simplification since the corresponding Faddeev-Popov determinant is trivial. Therefore, we set $(e, \chi, \bar{\chi}, a)=(T, \theta, \bar{\theta}, 0)$ to get

$$
\begin{aligned}
\int_{0}^{\infty} d T \int d \theta d \bar{\theta} \int_{\psi(T)=\Gamma}^{\bar{\psi}(T)=\bar{\Gamma}} \mathcal{D} \psi \mathcal{D} \bar{\psi} \\
\int_{x(0)=x_{i}}^{p(T)=p_{f}} \mathcal{D} x \mathcal{D} p e^{i p(T) \cdot x(T)-i \int_{0}^{T} d t\left(p \cdot \dot{x}+i \bar{\psi} \cdot \dot{\psi}-Q_{0}-\frac{\bar{\theta}}{T} Q_{1}-\frac{\theta}{T} Q_{2}-i \epsilon\right),}
\end{aligned}
$$

which can be compared with the analogous expression for the Dirac case of eq. (3.10).

At this point, a simple dimensional analysis reveals that eq. (4.21) cannot yield the propagator for a vector boson field $A_{\mu}(x)$, since the Grassmann integrals yield $Q_{1} Q_{2} / Q_{0}$, which behaves as $\sim p^{\mu} p^{\nu} / p^{2}$. In fact, the worldline representation of the $N=2$ model returns the propagator for the field strength tensor $F_{\mu \nu}$, rather than the fundamental field $A_{\mu}$. This property, which is well-known $[8,9,18,19,118]$ and shared by all models with $N$-extended supersymmetry with $N \geq 2$, should come as no surprise. It could have been guessed by the fact that the physical states corresponding to the quantization of the $N$-extended model in eq. (1.2) are constructed by taking the tensor product of the spin variables $\psi_{\mu}^{i}$, which correspond to the reducible Dirac representation $\left(\frac{1}{2}, 0\right) \oplus\left(0, \frac{1}{2}\right)$ of the Lorentz group. However, one could argue that for our purposes this is not a huge problem, since the underlying idea behind the representation of a dressed propagator for spin $1 / 2$ is that what matters for the asymptotic dynamics is the denominator contribution, and not the numerator.

In fact, the main obstacles appear when introducing a background gauge field $[12,21$, 119]. One might be tempted to do so by proceeding as we did for the spin 0 and spin $1 / 2$ cases, and replace the free charges $Q_{i}$ with the corresponding $Q_{i}^{A}$, where $p_{\mu} \rightarrow p_{\mu}+g \tilde{A}_{\mu}$. However, the equations of motion become inconsistent unless the field strength $\tilde{F}^{\mu \nu}$ of the background field is constant [120]. Moreover, a simple calculation reveals that the corresponding supersymmetry would require a vanishing $\tilde{F}^{\mu \nu}$. Once again, this problem is shared by all models with $N \geq 2$, and is related to the consistency problems of theories with charged fields of spin higher than $1 / 2[9,11]$. However, we can assume that for a soft background field the above conditions are approximately fulfilled. In fact, if $\tilde{A}_{\mu}$ is dominated by long wavelength components, the field strength is of order $k_{\mu} \tilde{A}_{\mu}$ and thus is subleading w.r.t. the hard momentum $p_{f}$ in the Lagrangian of eq. (4.21). Hence, supersymmetry is "softly" broken, and we can repeat the previous analysis carried in section 3. Let us discuss this in more detail.

The fact that the background field strength vanishes in the soft limit implies that eq. (4.21) can be regarded as the expectation value of the (approximately conserved) Noether charges $Q_{1}^{A}$ and $Q_{2}^{A}$, which can be evaluated at an arbitrary time. Again, for 
the given boundary conditions, the proper time $T$ is a convenient choice which yields

$$
Q_{1}^{A}(x(T), p(T))=\Gamma \cdot\left(p_{f}-A(x(T))\right), \quad Q_{2}^{A}(x(T), p(T))=\bar{\Gamma} \cdot\left(p_{f}-A(x(T))\right) .
$$

Plugging this into eq. (4.21) and performing the momentum integration around the classical solutions of eq. (2.8), we get

$$
\begin{aligned}
\left\langle p_{f} \mid x_{i}\right\rangle^{-1} & \left\langle p_{f}\left|\frac{Q_{1}^{A} Q_{2}^{A}}{2 Q_{0}^{A}+i \epsilon}\right| x_{i}\right\rangle= \\
& \frac{1}{2} \int_{0}^{\infty} d T e^{\frac{i}{2}\left(p_{f}^{2}+i \epsilon\right) T} \int d \theta d \bar{\theta} e^{i\left(\Gamma \cdot p_{f} \theta+\bar{\Gamma} \cdot p_{f} \theta\right)} f\left(x_{i}, p_{f}, \Gamma, \bar{\Gamma}, T, \theta, \bar{\theta}\right),
\end{aligned}
$$

where, in analogy with eq. (2.16) and eq. (3.16), we have defined

$$
\begin{aligned}
f\left(x_{i}, p_{f}, \Gamma, \bar{\Gamma}, T, \theta, \bar{\theta}\right)= & \int_{\psi(T)=\Gamma, \bar{\psi}(T)=\bar{\Gamma}} \mathcal{D} \psi \mathcal{D} \bar{\psi} \\
& \int_{\tilde{x}(0)=0} \mathcal{D} \tilde{x} e^{-i \theta \psi \cdot A\left(x_{i}+p_{f} T+\tilde{x}(T)\right)-i \bar{\theta} \bar{\psi} \cdot A\left(x_{i}+p_{f} T+\tilde{x}(T)\right)} \\
& \exp \left(i \int _ { 0 } ^ { T } d t \left(-i \bar{\psi} \cdot \dot{\psi}+\frac{1}{2} \dot{\tilde{x}}^{2}+\left(p_{f}+\dot{\tilde{x}}\right) \cdot A\left(x_{i}+p_{f} t+\tilde{x}(t)\right)\right.\right. \\
& \left.\left.+\frac{i}{2} \partial \cdot A\left(x_{i}+p_{f} t+\tilde{x}(t)\right)+\bar{\psi}_{\mu} \psi_{\nu} F^{\mu \nu}\right)\right) .
\end{aligned}
$$

At this point we should implement the same manipulations that we performed in the scalar and the Dirac cases, and truncate the external free propagator. However, in this case it means that we have to divide by the free correlator $\left\langle F_{\mu \nu} F_{\rho \sigma}\right\rangle$. Then, in analogy with eq. (3.17) and eq. (3.18), we consider the asymptotic limit $T \rightarrow \infty$ of eq. (4.24). The effect of this limit is that the background field in the first line of eq. (4.24) is evaluated for asymptotic times and thus it can be set to zero, so that the dependence of radiative factor $f\left(x_{i}, p_{f}, \Gamma, \bar{\Gamma}, T, \theta, \bar{\theta}\right)$ on $\theta$ and $\bar{\theta}$ can be dropped. Subsequently, the Grassmann integration over $\theta$ and $\bar{\theta}$ in eq. (4.23) becomes trivial and yields the prefactor $\Gamma \cdot p_{f} \bar{\Gamma} \cdot p_{f}$. This can be related to the numerator of the free correlator $\left\langle F_{\mu \nu} F_{\rho \sigma}\right\rangle$, once a suitable representation for the constant $\Gamma$ and $\bar{\Gamma}$ in terms of the gamma matrices is provided $[9,18]$.

To summarize, by studying the worldline $N=2$ model with the boundary conditions suitably chosen to describe the asymptotic dynamics, we obtained that the correlator $\left\langle F_{\mu \nu} F_{\rho \sigma}\right\rangle$ in the presence of a an asymptotic soft background field can be expressed in terms of the radiative factor

$$
\begin{aligned}
f\left(x_{i}, p_{f}, \Gamma, \bar{\Gamma}, \infty\right)= & \int_{\psi(\infty)=\Gamma, \bar{\psi}(\infty)=\bar{\Gamma}} \mathcal{D} \psi \mathcal{D} \bar{\psi} \int_{\tilde{x}(0)=0} \mathcal{D} \tilde{x} \\
& \exp \left(i \int _ { 0 } ^ { T } d t \left(-i \bar{\psi} \cdot \dot{\psi}+\frac{1}{2} \dot{\tilde{x}}^{2}+\left(p_{f}+\dot{\tilde{x}}\right) \cdot A\left(x_{i}+p_{f} t+\tilde{x}(t)\right)\right.\right. \\
& \left.\left.+\frac{i}{2} \partial \cdot A\left(x_{i}+p_{f} t+\tilde{x}(t)\right)+\bar{\psi}_{\mu} \psi_{\nu} F^{\mu \nu}\left(x_{i}+p_{f} t+\tilde{x}(t)\right)\right)\right) .
\end{aligned}
$$


Now we can solve the path integral order by order in the soft expansion by performing the usual rescaling $p_{f}^{\mu} \rightarrow \lambda n^{\mu}$ and $t \rightarrow t / \lambda$. We start with the Grassmann integrations, and expand around the boundary conditions

$$
\psi^{\mu}(t)=\psi^{\mu}(T)+\chi^{\mu}(t), \quad \bar{\psi}^{\mu}(t)=\bar{\psi}^{\mu}(T)+\bar{\chi}^{\mu}(t) .
$$

Then, the path integral becomes

$$
e^{\frac{i}{\lambda} \int_{0}^{\infty} d t \bar{\Gamma}_{\mu} \Gamma_{\nu} F^{\mu \nu}\left(x_{i}+p_{f} t+\tilde{x}(t)\right)} \int_{\chi(\infty)=0}^{\bar{\chi}(\infty)=0} \mathcal{D} \chi \mathcal{D} \bar{\chi} e^{i \int_{0}^{\infty} d t\left(-i \bar{\chi} \cdot \dot{\chi}+\frac{1}{\lambda} \bar{\chi}_{\mu} \chi_{\nu} F^{\mu \nu}\left(x_{i}+p_{f} t+\tilde{x}(t)\right)\right)} .
$$

Once again, the argument of $F^{\mu \nu}$ significantly simplifies by noting that $x_{i}^{\mu} \neq 0$ is a subleading effect, and that vertices with powers of $\tilde{x}^{\mu}$ and $\chi^{\mu}$ would require additional $\tilde{x}_{-}$ propagators, which are suppressed in $1 / \lambda$. This means that we can expand $F^{\mu \nu}$ at leading order in $\tilde{x}^{\mu}$, to get

$$
e^{\frac{i}{\lambda} \int_{0}^{\infty} d t \bar{\Gamma}_{\mu} \Gamma_{\nu} F^{\mu \nu}(n t)} \int_{\chi(\infty)=\bar{\chi}(\infty)=0} \mathcal{D} \chi \mathcal{D} \bar{\chi} e^{i \int_{0}^{\infty} d t\left(-i \bar{\chi} \cdot \dot{\chi}+\frac{1}{\lambda} \bar{\chi} \mu \chi_{\nu} F^{\mu \nu}(n t)\right)} .
$$

In analogy with section 3.3, the path integral is Gaussian and can be reabsorbed in the overall normalization with the factor $\left\langle p_{f} \mid x_{i}\right\rangle$ of eq. (4.23). The prefactor, on the other hand, contains boundary information in the term $\left[\bar{\Gamma}_{\mu}, \Gamma_{\nu}\right]$. With a suitable representation in terms of gamma matrices $[9,12,18]$, this returns once again the Lorentz generator $\left(S_{\mu \nu}\right)^{\rho \sigma}$, in agreement with eq. (4.14) and eq. (4.15).

Therefore, we recover the same structure of the previous section where the GWL has been derived without worldline fermions. Thus, despite the fact that the $N=2$ model corresponds to the propagation of the field strength $F^{\mu \nu}$ rather than the potential $A_{\mu}$, the denominator contribution is still given by a scalar term and a spin dependent term that involves the Lorentz generator $\left(S_{\mu \nu}\right)^{\rho \sigma}$. This is in agreement with the analogous result obtained with a one-loop effective action [12], thus confirming that the GWL is an equivalent description where only the denominator of a dressed propagator contributes to the asymptotics.

It is clear that the arguments presented in this section can be generalized to the case of a (massive) particle of arbitrary spin, thanks to the fact that the background field for $N>2$ is introduced in analogy with the $N=2$ case with a term proportional to $\psi_{i}^{\mu} \psi_{i}^{\nu} F^{\mu \nu}[11,120]$. Although this is not investigated further in this work, the term $\psi_{i}^{\mu} \psi_{i}^{\nu}$ gives rise to the corresponding Lorentz generator, once a proper representation in terms of gamma matrices is provided [8]. Therefore, the derivation is similar to the spin-one case, where the numerator is composed of quasi-conserved and effectively free Noether charges, while the contribution of the background field in the denominator is coupled to the corresponding Lorentz generator.

\section{Discussion}

The Generalized Wilson Line, originally proposed in [28] to extend the exponentiation of infrared radiation to next-to-leading power (NLP) and subsequently applied in $[71,72]$ to 
derive factorization theorems, is a powerful tool to describe asymptotic states dressed by soft radiation at subleading orders in the soft expansion. The derivation proposed in [28] concerned essentially the case of a scalar particle dressed by next-to-soft radiation, both in the abelian and in the non-abelian case. Although an argument for an extension to the case of spin $1 / 2$ particles was presented there, some issue remained to be clarified, while no proof was given for spin 1 or higher.

In this work, building on the well-known supersymmetric model for a spinning particle on the worldline, we have revisited the derivation for spin $1 / 2$. In particular, we have shown that the contribution of the soft background field to the numerator of an asymptotic dressed propagator vanishes. This fact, which was tacitly assumed in [28], is crucial to prove the truncation of the external free propagators in a scattering amplitude and was proven here by exploiting the supersymmetry of the corresponding worldline model.

Then, we considered the spin 1 case. Thanks to the gauge invariance of a scattering amplitude, a shortcut can be used in Feynman gauge, where the numerator is unity: since no background field appears in the numerator, no supersymmetric model is necessary and the derivation closely follows the scalar case. This implies that the next-to-eikonal exponentiation presented in $[28,33]$ and the related diagrammatic analysis of webs [31, 32] can be naturally applied to Yang-Mills theory.

Finally, we discussed how the GWL can be derived for particles of higher spin, by studying the $N=2$ wordline supersymmetric model. The obstacle here is that this model naturally describes the propagation of $F_{\mu \nu}$ rather than the fundamental field $A_{\mu}$. Moreover, a general background field is not compatible with wordline supersymmetry. However, although the derivation of the GWL is more challenging in this case, we presented an argument based on the observation that the field strength for the background field vanishes in the soft limit and that worldline supersymmetry is only softly broken.

For both the spin $1 / 2$ and spin 1 cases, the denominator contribution to the dressed propagator matches the one corresponding to the one-loop effective action [12], where the first-quantized Hamiltonian is given by the squared coviariant derivative $D^{2}$ plus a spin dependent term $S_{\mu \nu} F^{\mu \nu}$ representing the interaction between the magnetic moment of the emitting particle and the background soft field. For a single soft emission, this result was shown in [73] to be in agreement with the so-called tree-level next-to-soft theorems [55]. Although the GWL extends this statement to an arbitrary number of emissions, one must be careful in applying these technique in a scattering amplitude beyond the tree-level when massless particles are present or when the mass of the emitters is much smaller than the energy scale of the process, since it is well-known that collinear effects are not captured by this description alone and must be compensated by radiative jets [40, 52, 53, 71].

The analogy with the one-loop effective action is not surprising: as remarked in the introduction, at the cross-section level the external lines close at infinity, so it is natural to expect that the denominators, which do contribute to the asymptotic dynamics, must be the same for the closed and open dressed propagators. What we have shown in this work is that the GWL makes this picture valid at the amplitude level. This corroborates the intuitive idea that asymptotic propagators are a somewhat intermediate case between the closed and the open topologies. In fact, although the worldine formalism has been 
known for some time, the quantization of supersymmetric actions for spinning particles on the open topology is a relatively unexplored area, which might offer new insights into the structure of scattering amplitudes. The GWL offers a complementary point of view in this direction.

Although this paper has focused on the description of asymptotic states of a scattering amplitude dressed by soft photons or soft gluons, most of the ideas carries over to gravity theories. In fact, the gravitational GWL for scalar emitters has been already presented in [34], where the exponentiation of next-to-soft gravitons propagating in flat spacetime is discussed. In particular, defining the graviton $h^{\mu \nu}$ via $\sqrt{g} g^{\mu \nu}=\eta^{\mu \nu}+\kappa h^{\mu \nu}$, where $\kappa^{2}=32 \pi G$, one can treat $h^{\mu \nu}$ as a classical background and derive a consistent next-tosoft representation of the dressed scalar propagator with an expansion up to $\kappa^{2}$. This leads to a single-particle Hamiltonian of the form

$$
H=\frac{1}{2}\left(p^{2}-m^{2}\right)+\kappa \mathcal{F}\left(p^{\mu}, h^{\mu \nu}\right),
$$

where the presence of the background graviton field has been collected into a functional $\mathcal{F}\left(p^{\mu}, h^{\mu \nu}\right)$. As we can see, eq. (5.1) is the generalization of eq. (2.3) to gravitational interactions. The subsequent derivation is essentially the same of the one outlined in section 2 , even though the algebra is more involved, and it leads to the following structure for the gravitational GWL:

$$
\begin{aligned}
\widetilde{W}_{n}(0, \infty)=\exp [\kappa & \kappa \frac{d^{d} k}{(2 \pi)^{d}} \tilde{h}_{\mu \nu}(k) V^{\mu \nu}(p, k) \\
& \left.+\kappa^{2} \int \frac{d^{d} k}{(2 \pi)^{d}} \int \frac{d^{d} l}{(2 \pi)^{d}} \tilde{h}_{\mu \nu}(k) \tilde{h}_{\rho \sigma}(l) V^{\mu \nu \rho \sigma}(p, k, l)\right],
\end{aligned}
$$

where $\tilde{h}_{\mu \nu}$ is the Fourier transform of the graviton field, while $V^{\mu \nu}(p, k)$ and $V^{\mu \nu \rho \sigma}(p, k, l)$ are respectively a single-graviton and a double-graviton vertices, whose expressions can be found in [34]. In particular, the single graviton vertex is in agreement with the tree-level soft theorems [73].

At this point one might wonder whether the gravitational GWL can be defined also for spinning emitters. Although a complete derivation is beyond the scope of this paper, a naive guess is that one has to modify eq. (5.1) by adding a spin-dependent term that includes the Riemann curvature tensor, in analogy with the gauge theory case where one adds the term $S_{\mu \nu} F^{\mu \nu}$. In fact, the consistency of the equations of motion with a gravitational background field can be obtained from the gauge theory ones by replacing the field strength $F^{\mu \nu}$ with $\left[\nabla^{\mu}, \nabla^{\nu}\right]$, where $\nabla^{\mu}$ is the gravitational covariant derivative [120]. Therefore, leaving out the discussion of potential algebraic complications, the derivation does not seem to pose huge obstacles, at least for the contributions to the denominator of the dressed propagator. The main difficulties arise when considering numerators, where the contribution of worldline fermions is more delicate than the gauge theory case. This can be seen e.g. in the spin $1 / 2$ case, where the presence of gamma matrices in curved spacetime requires a careful treatment of the weak field expansion in terms of the graviton field. Moreover, one has to cope with the same issues encountered in section 4 of this paper, i.e. 
the corresponding worldline supersymmetric theory with a background gravitational field becomes inconsistent for $N>2$ (see e.g. [120]), which prevents a naive application of the method to pure gravity theories. The softness of the background field presumably restores the consistency of such representation. Work in this direction is ongoing.

For both gauge theories and gravity, the use of worldline techniques in the study of asymptotic dynamics can be also analyzed in the light of the revived interest in the Faddeev-Kulish coherent states [77-87], where the GWL offers a natural way to extend the analysis at subleading power in the soft expansion. In particular, the recently proposed prescription to define an infrared-finite S-matrix [89] provides a modern derivation of the asymptotic Hamiltonian in terms of Wilson lines and effective field theory techniques. In this regard, it is noteworthy that the asymptotic Hamiltonian of the Faddeev-Kulish construction corresponds to the single-particle Hamiltonian of the worldline formalism, i.e. the inverse propagator dressed by soft radiation. Therefore, the GWL offers a nice semiclassical interpretation of the asymptotic dressed state and also a natural extension of this picture at subleading power that bypasses the long derivation of the subleading Lagrangian in the effective field theory. In fact, although at the amplitude level infrared divergences are controlled by the well-known LP Wilson line operators, the situation is different for individual gauge-dependent diagrams, where in dimensional regularization poles might correspond to NLP effects that are controlled by the GWL. Moreover, the emergence of the spin-dependence at NLP has a very clear origin in the GWL description and it shows how it affects the asymptotics dynamics. Besides, the quantization of the asymptotic states emerges quite neatly in this picture, since the Hamiltonian is derived form the standard Dirac procedure to deal with constraints.

The semiclassical description of the GWL can shed light also on the logarithmic corrections to classical next-to-soft theorems in gauge and gravity theories, recently discussed in $[68,69,100]$. At the quantum level, the origin of such corrections is clear e.g. in dimensional regularization: loop corrections contains infrared poles which generate logarithms of the various scales involved the process. For particular configurations of the virtual particles, these scales contain the soft momentum $k$ and generate $\log (k)$, in contrast with the treelevel soft theorems [37, 38]. At the classical level one can trace back these logarithms to the long range forces that in four dimensions produce a logarithmic dependence on the proper time $T$ in the trajectory of the scattered particles. It is then argued that the corresponding contribution to soft theorems can be obtained by replacing $\log (T)$ with $\log (k)$. The GWL of this paper offers a nice bridge between the classical and the quantum description, being a tool to describe soft radiation of a quantum scattering amplitude as a perturbation of the classical path of the hard emitters. In particular, we have seen that the gauge field $A_{\mu}$ in eq. (2.16) (and similarly the gravitational field $h_{\mu \nu}$ in eq. (5.2)) acts as a source term on the worldline, generating fluctuations around the classical path of eq. (2.8). Assuming that the radiated field drops as $1 / t$ in the asymptotic limit, one could imagine to modify the classical trajectory by including a term $\sim x_{f}^{\mu} \log (t)$ due to the long range gravitational or electromagnetic force. In eq. (2.4) we have seen that a NLP contribution comes from having $x_{i}^{\mu} \neq 0$ in eq. (2.20) (from Low's theorem), which in turn gives the orbital angular momentum $L^{\mu \nu}$ dependence of the next-to-soft emission. Therefore, a $\log (t)$ term in the 
classical trajectory will give a logarithmic dependence in $L^{\mu \nu}$, in analogy with [68], hence in the exponent of the GWL, which corresponds to $\log (n \cdot k)$ in Fourier space.

The results presented in this work can be extended in many directions. The most obvious one is the generalization of the spin-one case to gluons in arbitrary gauges and to massive vector bosons. This problem would presumably need a worldline model where the spin variables take into account the different degrees of freedom that one wish to propagate, in analogy with the massive Dirac propagator that requires a fifth Grassmann variable. A second direction for future work is the generalization of the GWL to soft gravitons for spinning emitters, as already observed. The growing demand for precision calculations in gravitational physics, and the crucial role that spin effects might have to this aim [101111], make it natural to pursue this direction. Another aspect which is left for future work is a derivation of the GWL where the non-abelian nature of the soft background field is incorporated with additional Grassmann variables, on the line of the results obtained in the scalar case $[24,121]$. In this regard, it would be interesting to investigate what is the role of such variables in the soft expansion. Finally, there is growing evidence that the eikonal approximation underlies the classical limit of quantum scattering amplitudes [9096]. Hence, a comparison of the GWL for gauge bosons and gravitons might shed light on the worldline realization of the classical double copy [122], which has been recently investigated in [123-128].

\section{Acknowledgments}

The author would like to thank Fiorenzo Bastianelli, Anna Kulesza, Eric Laenen and Chris White for stimulating discussions.

Open Access. This article is distributed under the terms of the Creative Commons Attribution License (CC-BY 4.0), which permits any use, distribution and reproduction in any medium, provided the original author(s) and source are credited.

\section{References}

[1] J.S. Schwinger, On gauge invariance and vacuum polarization, Phys. Rev. 82 (1951) 664 [INSPIRE].

[2] C. Schubert, Perturbative quantum field theory in the string inspired formalism, Phys. Rept. 355 (2001) 73 [hep-th/0101036] [INSPIRE].

[3] J.P. Edwards and C. Schubert, Quantum mechanical path integrals in the first quantised approach to quantum field theory, arXiv:1912.10004 [INSPIRE].

[4] A. Barducci, R. Casalbuoni and L. Lusanna, Supersymmetries and the Pseudoclassical Relativistic electron, Nuovo Cim. A 35 (1976) 377 [InSPIRE].

[5] L. Brink, S. Deser, B. Zumino, P. Di Vecchia and P.S. Howe, Local Supersymmetry for Spinning Particles, Phys. Lett. B 64 (1976) 435 [Erratum ibid. 68 (1977) 488] [InSPIRE].

[6] L. Brink, P. Di Vecchia and P.S. Howe, A Lagrangian Formulation of the Classical and Quantum Dynamics of Spinning Particles, Nucl. Phys. B 118 (1977) 76 [InSPIRE]. 
[7] V.D. Gershun and V.I. Tkach, Classical and quantum dynamics of particles with arbitrary spin, JETP Lett. 29 (1979) 288 [inSPIRE].

[8] P.S. Howe, S. Penati, M. Pernici and P.K. Townsend, Wave Equations for Arbitrary Spin From Quantization of the Extended Supersymmetric Spinning Particle, Phys. Lett. B 215 (1988) 555 [INSPIRE].

[9] P.S. Howe, S. Penati, M. Pernici and P.K. Townsend, A Particle Mechanics Description of Antisymmetric Tensor Fields, Class. Quant. Grav. 6 (1989) 1125 [InSPIRE].

[10] E. Bergshoeff and J.W. van Holten, The Spectrum of Spinning Superparticles, Phys. Lett. B 226 (1989) 93 [INSPIRE].

[11] D.M. Gitman, A.E. Goncalves and I.V. Tyutin, Quantization of pseudoclassical model of spin one relativistic particle, Int. J. Mod. Phys. A 10 (1995) 701 [hep-th/9401132] [INSPIRE].

[12] M.J. Strassler, Field theory without Feynman diagrams: One loop effective actions, Nucl. Phys. B 385 (1992) 145 [hep-ph/9205205] [InSPIRE].

[13] Z. Bern and D.A. Kosower, The Computation of loop amplitudes in gauge theories, Nucl. Phys. B 379 (1992) 451 [INSPIRE].

[14] M.G. Schmidt and C. Schubert, On the calculation of effective actions by string methods, Phys. Lett. B 318 (1993) 438 [hep-th/9309055] [INSPIRE].

[15] M.G. Schmidt and C. Schubert, Worldline Green functions for multiloop diagrams, Phys. Lett. B 331 (1994) 69 [hep-th/9403158] [INSPIRE].

[16] P. Di Vecchia and F. Ravndal, Supersymmetric Dirac particles, Phys. Lett. A 73 (1979) 371 [INSPIRE].

[17] E.S. Fradkin and D.M. Gitman, Path integral representation for the relativistic particle propagators and BFV quantization, Phys. Rev. D 44 (1991) 3230 [INSPIRE].

[18] M. Pierri and V.O. Rivelles, BRST Quantization of Spinning Relativistic Particles With Extended Supersymmetries, Phys. Lett. B 251 (1990) 421 [INSPIRE].

[19] R. Marnelius, Proper BRST quantization of relativistic particles, Nucl. Phys. B 418 (1994) 353 [hep-th/9309002] [INSPIRE].

[20] J.W. van Holten, Propagators and path integrals, Nucl. Phys. B 457 (1995) 375 [hep-th/9508136] [INSPIRE].

[21] M. Reuter, M.G. Schmidt and C. Schubert, Constant external fields in gauge theory and the spin 0, 1/2, 1 path integrals, Annals Phys. 259 (1997) 313 [hep-th/9610191] [INSPIRE].

[22] C. Alexandrou, R. Rosenfelder and A.W. Schreiber, Worldline path integral for the massive Dirac propagator: a four-dimensional approach, Phys. Rev. A 59 (1999) 1762 [hep-th/9809101] [INSPIRE].

[23] P. Dai, Y.-t. Huang and W. Siegel, Worldgraph Approach to Yang-Mills Amplitudes from $N=2$ Spinning Particle, JHEP 10 (2008) 027 [arXiv:0807.0391] [InSPIRE].

[24] N. Ahmadiniaz, F. Bastianelli and O. Corradini, Dressed scalar propagator in a non-Abelian background from the worldline formalism, Phys. Rev. D 93 (2016) 025035 [Addendum ibid. 93 (2016) 049904] [arXiv: 1508.05144] [INSPIRE].

[25] S. Bhattacharya, Worldline Path-Integral Representations for Standard Model Propagators and Effective Actions, Adv. High Energy Phys. 2017 (2017) 2165731 [INSPIRE]. 
[26] N. Ahmadiniaz, V.M. Banda Guzmán, F. Bastianelli, O. Corradini, J.P. Edwards and C. Schubert, Worldline master formulas for the dressed electron propagator. Part I. Off-shell amplitudes, JHEP 08 (2020) 049 [arXiv: 2004.01391] [INSPIRE].

[27] O. Corradini and G.D. Esposti, Dressed Dirac Propagator from a Locally Supersymmetric $\mathcal{N}=1$ Spinning Particle, arXiv:2008.03114 [INSPIRE].

[28] E. Laenen, G. Stavenga and C.D. White, Path integral approach to eikonal and next-to-eikonal exponentiation, JHEP 03 (2009) 054 [arXiv:0811.2067] [INSPIRE].

[29] J.G.M. Gatheral, Exponentiation of Eikonal Cross-sections in Nonabelian Gauge Theories, Phys. Lett. B 133 (1983) 90 [InSPIRE].

[30] J. Frenkel and J.C. Taylor, Non abelian eikonal exponentiation, Nucl. Phys. B 246 (1984) 231 [INSPIRE].

[31] E. Gardi, E. Laenen, G. Stavenga and C.D. White, Webs in multiparton scattering using the replica trick, JHEP 11 (2010) 155 [arXiv:1008.0098] [INSPIRE].

[32] E. Gardi, J.M. Smillie and C.D. White, The Non-Abelian Exponentiation theorem for multiple Wilson lines, JHEP 06 (2013) 088 [arXiv:1304.7040] [INSPIRE].

[33] E. Laenen, L. Magnea, G. Stavenga and C.D. White, Next-to-Eikonal Corrections to Soft Gluon Radiation: A Diagrammatic Approach, JHEP 01 (2011) 141 [arXiv:1010.1860] [INSPIRE].

[34] C.D. White, Factorization Properties of Soft Graviton Amplitudes, JHEP 05 (2011) 060 [arXiv: 1103.2981] [INSPIRE].

[35] E. Laenen, L. Magnea, G. Stavenga and C.D. White, On Next-to-Eikonal Exponentiation, Nucl. Phys. B Proc. Suppl. 205-206 (2010) 260 [arXiv:1007.0624] [InSPIRE].

[36] S. Moch and A. Vogt, On non-singlet physical evolution kernels and large-x coefficient functions in perturbative QCD, JHEP 11 (2009) 099 [arXiv: 0909.2124] [INSPIRE].

[37] D. Bonocore, E. Laenen, L. Magnea, L. Vernazza and C.D. White, The method of regions and next-to-soft corrections in Drell-Yan production, Phys. Lett. B 742 (2015) 375 [arXiv: 1410.6406] [INSPIRE].

[38] D. Bonocore, E. Laenen, L. Magnea, S. Melville, L. Vernazza and C.D. White, $A$ factorization approach to next-to-leading-power threshold logarithms, JHEP 06 (2015) 008 [arXiv: 1503.05156] [INSPIRE].

[39] I. Moult, I.W. Stewart, G. Vita and H.X. Zhu, First Subleading Power Resummation for Event Shapes, JHEP 08 (2018) 013 [arXiv:1804.04665] [INSPIRE].

[40] I. Moult, I.W. Stewart and G. Vita, Subleading Power Factorization with Radiative Functions, JHEP 11 (2019) 153 [arXiv: 1905.07411] [INSPIRE].

[41] I. Moult, G. Vita and K. Yan, Subleading power resummation of rapidity logarithms: the energy-energy correlator in $\mathcal{N}=4$ SYM, JHEP 07 (2020) 005 [arXiv:1912.02188] [INSPIRE].

[42] M.A. Ebert, I. Moult, I.W. Stewart, F.J. Tackmann, G. Vita and H.X. Zhu, Power Corrections for N-Jettiness Subtractions at $\mathcal{O}\left(\alpha_{s}\right)$, JHEP 12 (2018) 084 [arXiv: 1807.10764] [INSPIRE].

[43] C. Anastasiou, C. Duhr, F. Dulat, F. Herzog and B. Mistlberger, Higgs Boson Gluon-Fusion Production in QCD at Three Loops, Phys. Rev. Lett. 114 (2015) 212001 [arXiv: 1503. 06056] [INSPIRE]. 
[44] N. Bahjat-Abbas, J. Sinninghe Damsté, L. Vernazza and C.D. White, On next-to-leading power threshold corrections in Drell-Yan production at $N^{3} L O$, JHEP 10 (2018) 144 [arXiv: 1807.09246] [INSPIRE].

[45] M. van Beekveld, W. Beenakker, R. Basu, E. Laenen, A. Misra and P. Motylinski, Next-to-leading power threshold effects for resummed prompt photon production, Phys. Rev. D 100 (2019) 056009 [arXiv: 1905.11771] [INSPIRE].

[46] M. van Beekveld, W. Beenakker, E. Laenen and C.D. White, Next-to-leading power threshold effects for inclusive and exclusive processes with final state jets, JHEP 03 (2020) 106 [arXiv: 1905.08741] [INSPIRE].

[47] V. Del Duca, E. Laenen, L. Magnea, L. Vernazza and C.D. White, Universality of next-to-leading power threshold effects for colourless final states in hadronic collisions, JHEP 11 (2017) 057 [arXiv:1706. 04018] [InSPIRE].

[48] M. Beneke, M. Garny, R. Szafron and J. Wang, Anomalous dimension of subleading-power $N$-jet operators, JHEP 03 (2018) 001 [arXiv:1712.04416] [inSPIRE].

[49] M. Beneke, M. Garny, R. Szafron and J. Wang, Anomalous dimension of subleading-power $N$-jet operators. Part II, JHEP 11 (2018) 112 [arXiv:1808.04742] [INSPIRE].

[50] M. Beneke, A. Broggio, M. Garny, S. Jaskiewicz, R. Szafron, L. Vernazza et al., Leading-logarithmic threshold resummation of the Drell-Yan process at next-to-leading power, JHEP 03 (2019) 043 [arXiv:1809.10631] [INSPIRE].

[51] M. Beneke, A. Broggio, S. Jaskiewicz and L. Vernazza, Threshold factorization of the Drell-Yan process at next-to-leading power, JHEP 07 (2020) 078 [arXiv:1912.01585] [INSPIRE].

[52] H. Gervais, Soft Photon Theorem for High Energy Amplitudes in Yukawa and Scalar Theories, Phys. Rev. D 95 (2017) 125009 [arXiv:1704.00806] [InSPIRE].

[53] E. Laenen, J. Sinninghe Damsté, L. Vernazza, W. Waalewijn and L. Zoppi, Towards all-order factorization of QED amplitudes at next-to-leading power, arXiv:2008.01736 [INSPIRE].

[54] A. Strominger, On BMS Invariance of Gravitational Scattering, JHEP 07 (2014) 152 [arXiv: 1312.2229] [INSPIRE].

[55] F. Cachazo and A. Strominger, Evidence for a New Soft Graviton Theorem, arXiv: 1404.4091 [INSPIRE].

[56] E. Casali, Soft sub-leading divergences in Yang-Mills amplitudes, JHEP 08 (2014) 077 [arXiv: 1404.5551] [INSPIRE].

[57] Z. Bern, S. Davies and J. Nohle, On Loop Corrections to Subleading Soft Behavior of Gluons and Gravitons, Phys. Rev. D 90 (2014) 085015 [arXiv:1405.1015] [InSPIRE].

[58] A.J. Larkoski, D. Neill and I.W. Stewart, Soft Theorems from Effective Field Theory, JHEP 06 (2015) 077 [arXiv: 1412.3108] [INSPIRE].

[59] S. He, Y.-t. Huang and C. Wen, Loop Corrections to Soft Theorems in Gauge Theories and Gravity, JHEP 12 (2014) 115 [arXiv:1405.1410] [INSPIRE].

[60] A.J. Larkoski, Conformal Invariance of the Subleading Soft Theorem in Gauge Theory, Phys. Rev. D 90 (2014) 087701 [arXiv:1405.2346] [INSPIRE].

[61] N. Afkhami-Jeddi, Soft Graviton Theorem in Arbitrary Dimensions, arXiv:1405.3533 [INSPIRE]. 
[62] T. Adamo, E. Casali and D. Skinner, Perturbative gravity at null infinity, Class. Quant. Grav. 31 (2014) 225008 [arXiv: 1405.5122] [INSPIRE].

[63] T. He, V. Lysov, P. Mitra and A. Strominger, BMS supertranslations and Weinberg's soft graviton theorem, JHEP 05 (2015) 151 [arXiv:1401.7026] [INSPIRE].

[64] T. Klose, T. McLoughlin, D. Nandan, J. Plefka and G. Travaglini, Double-Soft Limits of Gluons and Gravitons, arXiv:1504.05558 [INSPIRE].

[65] A. Brandhuber, E. Hughes, B. Spence and G. Travaglini, One-Loop Soft Theorems via Dual Superconformal Symmetry, JHEP 03 (2016) 084 [arXiv: 1511.06716] [INSPIRE].

[66] A. Sen, Subleading Soft Graviton Theorem for Loop Amplitudes, JHEP 11 (2017) 123 [arXiv: 1703.00024] [INSPIRE].

[67] A. Laddha and A. Sen, Sub-subleading Soft Graviton Theorem in Generic Theories of Quantum Gravity, JHEP 10 (2017) 065 [arXiv: 1706.00759] [INSPIRE].

[68] B. Sahoo and A. Sen, Classical and Quantum Results on Logarithmic Terms in the Soft Theorem in Four Dimensions, JHEP 02 (2019) 086 [arXiv:1808.03288] [INSPIRE].

[69] A. Laddha and A. Sen, Logarithmic Terms in the Soft Expansion in Four Dimensions, JHEP 10 (2018) 056 [arXiv:1804.09193] [INSPIRE].

[70] B. Sahoo, Classical Sub-subleading Soft Photon and Soft Graviton Theorems in Four Spacetime Dimensions, JHEP 12 (2020) 070 [arXiv: 2008. 04376] [INSPIRE].

[71] D. Bonocore, E. Laenen, L. Magnea, L. Vernazza and C.D. White, Non-abelian factorisation for next-to-leading-power threshold logarithms, JHEP 12 (2016) 121 [arXiv: 1610.06842] [INSPIRE].

[72] N. Bahjat-Abbas, D. Bonocore, J. Sinninghe Damsté, E. Laenen, L. Magnea, L. Vernazza et al., Diagrammatic resummation of leading-logarithmic threshold effects at next-to-leading power, JHEP 11 (2019) 002 [arXiv:1905.13710] [INSPIRE].

[73] C.D. White, Diagrammatic insights into next-to-soft corrections, Phys. Lett. B 737 (2014) 216 [arXiv: 1406.7184] [INSPIRE].

[74] P.P. Kulish and L.D. Faddeev, Asymptotic conditions and infrared divergences in quantum electrodynamics, Theor. Math. Phys. 4 (1970) 745 [INSPIRE].

[75] S. Catani, M. Ciafaloni and G. Marchesini, Noncancelling infrared divergences in QCD coherent state, Nucl. Phys. B 264 (1986) 588 [inSPIRE].

[76] V. Del Duca, L. Magnea and G.F. Sterman, Collinear Infrared Factorization and Asymptotic Evolution, Nucl. Phys. B 324 (1989) 391 [INSPIRE].

[77] D. Kapec, M. Perry, A.-M. Raclariu and A. Strominger, Infrared Divergences in QED, Revisited, Phys. Rev. D 96 (2017) 085002 [arXiv: 1705.04311] [INSPIRE].

[78] A. Strominger, Lectures on the Infrared Structure of Gravity and Gauge Theory, arXiv: 1703.05448 [INSPIRE].

[79] S. Pasterski and S.-H. Shao, Conformal basis for flat space amplitudes, Phys. Rev. D 96 (2017) 065022 [arXiv:1705.01027] [INSPIRE].

[80] D. Carney, L. Chaurette, D. Neuenfeld and G. Semenoff, On the need for soft dressing, JHEP 09 (2018) 121 [arXiv:1803.02370] [INSPIRE].

[81] R. Gonzo, T. Mc Loughlin, D. Medrano and A. Spiering, Asymptotic Charges and Coherent States in $Q C D$, arXiv:1906.11763 [INSPIRE]. 
[82] S. Choi and R. Akhoury, Subleading soft dressings of asymptotic states in QED and perturbative quantum gravity, JHEP 09 (2019) 031 [arXiv:1907.05438] [INSPIRE].

[83] M. Pate, A.-M. Raclariu, A. Strominger and E.Y. Yuan, Celestial Operator Products of Gluons and Gravitons, arXiv:1910.07424 [INSPIRE].

[84] H. Hirai and S. Sugishita, Dressed states from gauge invariance, JHEP 06 (2019) 023 [arXiv: 1901.09935] [INSPIRE].

[85] Y.T.A. Law and M. Zlotnikov, Massive Spinning Bosons on the Celestial Sphere, JHEP 06 (2020) 079 [arXiv: 2004.04309] [INSPIRE].

[86] E. Casali and A. Puhm, A Double Copy for Celestial Amplitudes, arXiv:2007.15027 [INSPIRE].

[87] S.A. Narayanan, Massive Celestial Fermions, JHEP 12 (2020) 074 [arXiv: 2009.03883] [INSPIRE].

[88] H. Hannesdottir and M.D. Schwartz, A Finite S-Matrix, arXiv:1906.03271 [INSPIRE].

[89] H. Hannesdottir and M.D. Schwartz, S-Matrix for massless particles, Phys. Rev. D 101 (2020) 105001 [arXiv:1911.06821] [INSPIRE].

[90] D.A. Kosower, B. Maybee and D. O'Connell, Amplitudes, Observables, and Classical Scattering, JHEP 02 (2019) 137 [arXiv: 1811.10950] [INSPIRE].

[91] B. Maybee, D. O'Connell and J. Vines, Observables and amplitudes for spinning particles and black holes, JHEP 12 (2019) 156 [arXiv:1906.09260] [INSPIRE].

[92] L. de la Cruz, B. Maybee, D. O'Connell and A. Ross, Classical Yang-Mills observables from amplitudes, JHEP 12 (2020) 076 [arXiv:2009.03842] [INSPIRE].

[93] D. Amati, M. Ciafaloni and G. Veneziano, Superstring Collisions at Planckian Energies, Phys. Lett. B 197 (1987) 81 [inSPIRE].

[94] T. Damour, High-energy gravitational scattering and the general relativistic two-body problem, Phys. Rev. D 97 (2018) 044038 [arXiv:1710.10599] [INSPIRE].

[95] M. Ciafaloni, D. Colferai and G. Veneziano, Infrared features of gravitational scattering and radiation in the eikonal approach, Phys. Rev. D 99 (2019) 066008 [arXiv:1812.08137] [INSPIRE].

[96] A. Koemans Collado, P. Di Vecchia and R. Russo, Revisiting the second post-Minkowskian eikonal and the dynamics of binary black holes, Phys. Rev. D 100 (2019) 066028 [arXiv: 1904.02667] [INSPIRE].

[97] Z. Bern, H. Ita, J. Parra-Martinez and M.S. Ruf, Universality in the classical limit of massless gravitational scattering, Phys. Rev. Lett. 125 (2020) 031601 [arXiv:2002.02459] [INSPIRE].

[98] S. Melville, S.G. Naculich, H.J. Schnitzer and C.D. White, Wilson line approach to gravity in the high energy limit, Phys. Rev. D 89 (2014) 025009 [arXiv:1306.6019] [InSPIRE].

[99] A. Luna, S. Melville, S.G. Naculich and C.D. White, Next-to-soft corrections to high energy scattering in QCD and gravity, JHEP 01 (2017) 052 [arXiv:1611.02172] [INSPIRE].

[100] M. A, D. Ghosh, A. Laddha and P.V. Athira, Soft Radiation from Scattering Amplitudes Revisited, arXiv: 2007.02077 [INSPIRE].

[101] M. Levi, Next to Leading Order gravitational Spin-Orbit coupling in an Effective Field Theory approach, Phys. Rev. D 82 (2010) 104004 [arXiv:1006.4139] [INSPIRE]. 
[102] M. Levi, Effective Field Theories of Post-Newtonian Gravity: A comprehensive review, Rept. Prog. Phys. 83 (2020) 075901 [arXiv: 1807.01699] [InSPIRE].

[103] M. Levi and J. Steinhoff, Leading order finite size effects with spins for inspiralling compact binaries, JHEP 06 (2015) 059 [arXiv: 1410.2601] [INSPIRE].

[104] M. Levi and J. Steinhoff, Next-to-next-to-leading order gravitational spin-squared potential via the effective field theory for spinning objects in the post-Newtonian scheme, JCAP 01 (2016) 008 [arXiv: 1506.05794] [INSPIRE].

[105] M. Levi, S. Mougiakakos and M. Vieira, Gravitational cubic-in-spin interaction at the next-to-leading post-Newtonian order, JHEP 01 (2021) 036 [arXiv:1912.06276] [INSPIRE].

[106] M. Levi, A.J. Mcleod and M. Von Hippel, $N^{3} L O$ gravitational spin-orbit coupling at order

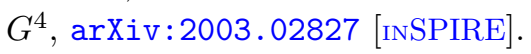

[107] J. Vines and J. Steinhoff, Spin-multipole effects in binary black holes and the test-body limit, Phys. Rev. D 97 (2018) 064010 [arXiv: 1606.08832] [InSPIRE].

[108] A. Guevara, A. Ochirov and J. Vines, Scattering of Spinning Black Holes from Exponentiated Soft Factors, JHEP 09 (2019) 056 [arXiv: 1812.06895] [INSPIRE].

[109] Z. Bern, A. Luna, R. Roiban, C.-H. Shen and M. Zeng, Spinning Black Hole Binary Dynamics, Scattering Amplitudes and Effective Field Theory, arXiv:2005.03071 [INSPIRE].

[110] A. Antonelli, C. Kavanagh, M. Khalil, J. Steinhoff and J. Vines, Gravitational spin-orbit coupling through third-subleading post-Newtonian order: from first-order self-force to arbitrary mass ratios, Phys. Rev. Lett. 125 (2020) 011103 [arXiv:2003.11391] [INSPIRE].

[111] A. Matas et al., Aligned-spin neutron-star-black-hole waveform model based on the effective-one-body approach and numerical-relativity simulations, Phys. Rev. D 102 (2020) 043023 [arXiv: 2004.10001] [INSPIRE].

[112] M. Bañados and I.A. Reyes, A short review on Noether's theorems, gauge symmetries and boundary terms, Int. J. Mod. Phys. D 25 (2016) 1630021 [arXiv:1601.03616] [InSPIRE].

[113] N. Agarwal, A. Danish, L. Magnea, S. Pal and A. Tripathi, Multiparton webs beyond three loops, JHEP 05 (2020) 128 [arXiv: 2003.09714] [INSPIRE].

[114] F.A. Berezin and M.S. Marinov, Particle Spin Dynamics as the Grassmann Variant of Classical Mechanics, Annals Phys. 104 (1977) 336 [INSPIRE].

[115] M.D. Schwartz, Quantum Field Theory and the Standard Model, Cambridge University Press, (2014).

[116] F. Bastianelli, P. Benincasa and S. Giombi, Worldline approach to vector and antisymmetric tensor fields. II, JHEP 10 (2005) 114 [hep-th/0510010] [INSPIRE].

[117] F. Bastianelli, P. Benincasa and S. Giombi, Worldline approach to vector and antisymmetric tensor fields, JHEP 04 (2005) 010 [hep-th/0503155] [INSPIRE].

[118] F. Bastianelli, O. Corradini and E. Latini, Higher spin fields from a worldline perspective, JHEP 02 (2007) 072 [hep-th/0701055] [INSPIRE].

[119] G.M. Shore, Symmetry Restoration and the Background Field Method in Gauge Theories, Annals Phys. 137 (1981) 262 [INSPIRE].

[120] I.L. Buchbinder and S.M. Shvartsman, Derivation of the actions for the relativistic particles with arbitrary spins, Int. J. Mod. Phys. A 8 (1993) 683 [INSPIRE]. 
[121] J.P. Edwards and O. Corradini, Mixed symmetry Wilson-loop interactions in the worldline formalism, JHEP 09 (2016) 081 [arXiv: 1607.04230] [INSPIRE].

[122] R. Monteiro, D. O'Connell and C.D. White, Black holes and the double copy, JHEP 12 (2014) 056 [arXiv: 1410.0239] [INSPIRE].

[123] W.D. Goldberger and A.K. Ridgway, Radiation and the classical double copy for color charges, Phys. Rev. D 95 (2017) 125010 [arXiv:1611.03493] [INSPIRE].

[124] D. Chester, Radiative double copy for Einstein-Yang-Mills theory, Phys. Rev. D 97 (2018) 084025 [arXiv: 1712.08684] [INSPIRE].

[125] C.-H. Shen, Gravitational Radiation from Color-Kinematics Duality, JHEP 11 (2018) 162 [arXiv: 1806. 07388] [INSPIRE].

[126] J. Plefka, C. Shi, J. Steinhoff and T. Wang, Breakdown of the classical double copy for the effective action of dilaton-gravity at NNLO, Phys. Rev. D 100 (2019) 086006 [arXiv: 1906. 05875] [INSPIRE].

[127] A.P.V. and A. Manu, Classical double copy from Color Kinematics duality: A proof in the soft limit, Phys. Rev. D 101 (2020) 046014 [arXiv:1907.10021] [INSPIRE].

[128] G.L. Almeida, S. Foffa and R. Sturani, Classical Gravitational Self-Energy from Double Copy, JHEP 11 (2020) 165 [arXiv:2008.06195] [INSPIRE]. 